\title{
$W^{a} \vee$ e phenomena
}

\section{Polynomial stability for a system of coupled strings}

Łukasz Rzepnicki, Roland Schnaubelt

CRC Preprint 2017/35, December 2017

\section{KARLSRUHE INSTITUTE OF TECHNOLOGY}

\section{CRC 1173}

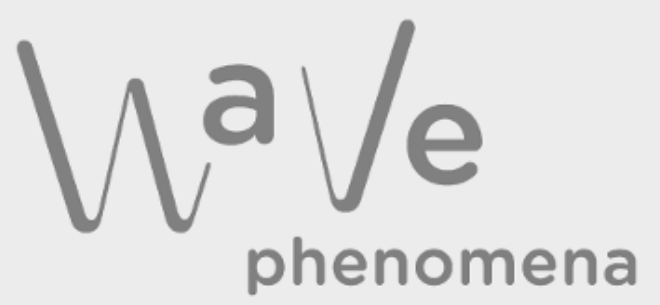




\section{Participating universities}

EBERHARD KARLS

UNIVERSITATT TUBINGEN

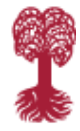

Funded by

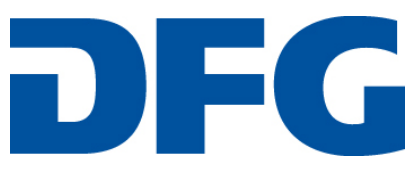

ISSN 2365-662X 


\title{
POLYNOMIAL STABILITY FOR A SYSTEM OF COUPLED STRINGS
}

\author{
ŁUKASZ RZEPNICKI AND ROLAND SCHNAUBELT
}

\begin{abstract}
We study the long-time behavior of two vibrating strings which are coupled at a common boundary point by a damper. We show that the classical solutions converge polynomially with a uniform rate, where the decay exponent depends on number theoretic properties of the quotient of the wave speeds of the two springs. The proof is based on a resolvent characterization of polynomial stability due to BorichevTomilov and Batty-Duyckaerts.
\end{abstract}

\section{INTRODUCTION}

In this paper we investigate the long-term behavior of two string equations which are coupled by a boundary damper, see (1.1) and (1.8). Their asymptotic properties heavily depend on the ratio $d=c_{2} / c_{1}$ of the wave speeds $c_{k}$ of the two strings. The case of rational $d$ was studied in [19] and [12]. In our main result Theorem 4.2 we show the polynomial convergence of all classical solutions to 0 , to a constant or to an at most linearly growing solution, respectively, depending on the chosen boundary and interface conditions. The convergence rate is determined by a irrationality measure of $d$, which describes how fast $d$ can be approximated by (appropriate) rational numbers, cf. Section 3. We also see that the obtained decay rate is almost sharp. Such diophantine properties of a parameter have been used before in the stability theory of evolution equations in e.g. [1], [14], [15] or [26], where other problems were treated by different methods. However, our system seems to be the first one exhibiting an explicit dependance of the (polynomial) decay on such number theoretic quantities.

In recent years there has been an important progress in the understanding of stability properties of linear evolution equations beyond the well studied case of exponential decay. In a Hilbert space, Gearhart's theorem characterizes the exponential convergence to 0 of all (mild) solutions by the boundedness of the resolvent $R(\lambda, A)$ for $\operatorname{Re} \lambda \geq 0$, where $A$ is the generator governing the problem. But damping mechanism in wave type equations often lead to weaker decay properties, see e.g. the discussion in [2].

1991 Mathematics Subject Classification. Primary: 35B40. Secondary: 35L53, 47D06.

Key words and phrases. Polynomial decay, boundary damping, resolvent bound, irrationality measure.

We thank Przemysław Berk, Yann Bugeaud, Stefan Kühnlein and Andrzej Schinzel for their help concerning questions about number theory. R.S. gratefully acknowledges financial support by the Deutsche Forschungsgemeinschaft (DFG) through CRC 1173. Ł. Rz. was partially supported by the National Science Centre (Poland) grant DEC2014/13/B/ST1/03153. 
Logarithmic decay of all classical solutions $u$ (i.e., $u(0) \in D(A)$ ) was characaterized in terms of resolvent estimates in the pioneering work [18]. For a bounded semigroup $T(\cdot)$ with generator $A$ on a Hilbert space $X$, one has the polynomial decay $\left\|T(t)(I-A)^{-1}\right\| \leq c t^{-1 / \alpha}$ for $t \geq 1$ and some $\alpha>0$ if and only if the spectrum of $A$ belongs to the open left half plane and $\left\|(i s-A)^{-1}\right\| \leq c|s|^{\alpha}$ for real $s$ with $|s| \geq 1$. The sufficiency of the conditions on $A$ was shown in [8], whereas their necessity is true even in a Banach space $X$ by [6]. (See [3] and [20] for earlier contributions.) The sufficiency fails in $L^{1}$-spaces in general, see [8]. Still in Banach spaces the resolvent condition implies the corresponding decay up to a logarithmic correction, as shown in [6] also for more general rates. In the recent papers [4] and [5] these results have been refined in various directions. In these papers one finds plenty of references concerning applications to PDEs, see also [2], [3], [6], [8], [20].

In this work we establish the polynomial decay of the system of two strings

$$
\begin{aligned}
\partial_{t t} u(t, x) & =c_{1}^{2} \partial_{x x} u(t, x), & & x \in(-1,0), \quad t \geq 0, \\
\partial_{t t} v(t, x) & =c_{2}^{2} \partial_{x x} v(t, x), & & x \in(0,1), \quad t \geq 0, \\
u(0, x) & =u_{0}(x), \quad \partial_{t} u(0, x)=u_{1}(x), & & x \in(-1,0), \\
v(0, x) & =v_{0}(x), \quad \partial_{t} v(0, x)=v_{1}(x), & & x \in(0,1),
\end{aligned}
$$

with the wave speeds $c_{k}=\sqrt{T_{k} / m_{k}}$ and initial functions $u_{0}, u_{1}, v_{0}, v_{1}$. The mass and tension densities $m_{1}, m_{2}>0$ and $T_{1}, T_{2}>0$ are given constants. In (1.8) we add to (1.1) some of the boundary and interface conditions

$$
\begin{aligned}
u(t,-1) & =0, \\
\partial_{x} u(t,-1) & =0, \\
v(t, 1) & =0, \\
\partial_{x} v(t, 1) & =0, \\
T_{1} \partial_{x} u(t, 0) & =T_{2} \partial_{x} v(t, 0), \quad \partial_{t} u(t, 0)-\partial_{t} v(t, 0)=-k T_{1} \partial_{x} u(t, 0), \\
u(t, 0) & =v(t, 0), \quad T_{1} \partial_{x} u(t, 0)-T_{2} \partial_{x} v(t, 0)=-k \partial_{t} u(t, 0),
\end{aligned}
$$

for $t \geq 0$ and a damping constant $k>0$.

This system was introduced in [11], where a mechanical interpretation of the interface damping was given and in particular the case $c_{1}=c_{2}$ was studied. The long-time behavior of this system was further investigated in [19]. There it was seen that the quotient $d=c_{2} / c_{1}$ plays a crucial role in the analysis of (1.1). If it is a rational number of the right type, see (2.19), then the energy of all solutions decays exponentially. For other rational numbers there are time periodic solutions and thus no decay. For irrational $d$, convergence of the solutions was shown without providing rates. Actually, the paper [19] contains some errors in details, partly fixed in [12], see Section 2.

Let $d$ be irrational. As in [19], we complement (1.1) by the combinations

$$
\begin{array}{lll}
\text { Case I : } & (1.2) \text { and }(1.4) \text { and (1.6), } \\
\text { Case II : } & (1.2) \text { and }(1.5) \text { and (1.6), } \\
\text { Case II : } & (1.3) \text { and }(1.5) \text { and (1.6), } \\
\text { Case IV : } & (1.2) \text { and (1.4) and (1.7), } \\
\text { Case V : } & (1.2) \text { and (1.5) and (1.7), } \\
\text { Case VI : } & (1.3) \text { and (1.5) and (1.7). }
\end{array}
$$


We note that the long-time behavior in case III was not studied in [19] and [12]. In the next section we see that in each case the system is governed by a generator $A_{j}$ on a suitable Hilbert space $\mathcal{H}_{j}$. In the cases I, IV and V the spectrum of $A_{j}$ belongs to the open left half plane, whereas in the other cases 0 is an isolated eigenvalue of $A_{j}$. More precisely, in II and VI we only have a nontrivial kernel of $A_{j}$, but $A_{I I I}$ also has a proper generalized eigenvector for $\lambda=0$. Accordingly, we show polynomial decay to 0 , respectively to a constant, for $j \neq I I I$, and to an at most linearly growing solution if $j=$ $I I I$, see Theorems 4.2 and 4.3. It is crucial for our approach to obtain the boundedness of the solution semigroup, respectively of its restriction to the kernel of the spectral projection for the spectral set $\{0\}$. This fact is fairly standard for $j \in\{I, I V, V\}$. For $j \in\{I I, V I\}$ one can use ideas from the recent paper [7] about a certain parabolic-hyperbolic system in one space dimension. The case III, however, requires a detailed analysis of the spectral projection, cf. Proposition 2.2.

The main results heavily depend on the decay behavior of a complex function $\Delta_{j}(i s)$ as $s \rightarrow \pm \infty$, see $(2.12)$, since the resolvent $\left(i s-A_{j}\right)^{-1}$ is bounded by $c\left|\Delta_{j}(i s)\right|^{-1}$ thanks to Proposition 2.2. The map $\Delta_{j}(i s)$ is a linear combination of products of $\sin \left(c_{k} s\right)$ and $\cos \left(c_{k} s\right)$ for $k \in\{1,2\}$. Therefore its decay is determined by the rate with which one can approximate $d$ by fractions $p / q$ of the type odd/odd, even/odd or odd/even, depending of the case $j$, see (3.2). We call the optimal rate $\mu_{j}(d)$, and it is closely related to the irrationality measure on $d$, which has thoroughly been studied in number theory as discussed in Section 3. One has $\mu_{j}(d) \geq 2$ for every irrational $d$ and equality holds if $d$ algebraic by Roth's theorem [22].

Depending on $\mu_{j}(d)$, we can then compute the decay rate of $\Delta_{j}(i s)$ to 0 in Lemma 3.1 in a rather delicate way where we crucially use the structure of $\Delta_{j}(i s)$. It is furrther shown that the obtained rate is almost optimal. Astonishingly one can discuss the six cases more or less in parallel. In the last section we then prove our main results, also using the results of [6] and [8]. For classical solutions, Theorem 4.2 gives almost optimal polynomial decay rates in terms of the irrationality measure of $d$. For instance, if $\mu_{j}(d)=2$, we have the decay $c_{\epsilon} t^{-\frac{1}{2}-\epsilon}$ for all $\epsilon>0$ and solutions starting in the unit ball of $D\left(A_{j}\right)$, and we know that this exponent is almost sharp. Based on somewhat different number theoretic results, in Theorem 4.3 we finally show decay by $t^{-1 / 2}$ times a logarithmic correction, for a.e. $d$.

\section{Basic PROPERTIES OF the SOlUtion SEMIGROUP}

We rewrite the system (1.1) with the boundary conditions in (1.8) as an evolution equation of first order in time for the states $\left(u, \partial_{t} u, v, \partial_{t} v\right)=w=$ $\left(w_{1}, w_{2}, w_{3}, w_{4}\right)$ in the product space

$$
\mathcal{H}=H^{1}(-1,0) \times L_{2}(-1,0) \times H^{1}(0,1) \times L_{2}(0,1)
$$

which is equipped with its canonical norm $\|\cdot\|_{\mathcal{H}}$. We mostly work in smaller state spaces which include the Dirichlet conditions from (1.8); namely in

$$
\begin{aligned}
\mathcal{H}_{I} & =\left\{w \in \mathcal{H} \mid w_{1}(-1)=0=w_{3}(1)\right\}, \\
\mathcal{H}_{I I} & =\left\{w \in \mathcal{H} \mid w_{1}(-1)=0\right\},
\end{aligned}
$$




$$
\begin{aligned}
\mathcal{H}_{I I I} & =\mathcal{H}, \\
\mathcal{H}_{I V} & =\left\{w \in \mathcal{H} \mid w_{1}(-1)=0=w_{3}(1), w_{1}(0)=w_{3}(0)\right\}, \\
\mathcal{H}_{V} & =\left\{w \in \mathcal{H} \mid w_{1}(-1)=0, w_{1}(0)=w_{3}(0)\right\}, \\
\mathcal{H}_{V I} & =\left\{w \in \mathcal{H} \mid w_{1}(0)=w_{3}(0)\right\}
\end{aligned}
$$

for the respective cases $j \in J:=\{I, I I, I I I, I V, V, V I\}$, noting that $H^{1}(a, b)$ embeds into $C([a, b])$. We endow $\mathcal{H}_{I}, \mathcal{H}_{I V}$, and $\mathcal{H}_{V}$ with the scalar product

$$
\langle w, z\rangle_{E}=\frac{1}{2} \int_{-1}^{0}\left(T_{1} w_{1}^{\prime} \bar{z}_{1}^{\prime}+m_{1} w_{2} \bar{z}_{2}\right) \mathrm{d} x+\frac{1}{2} \int_{0}^{1}\left(T_{2} w_{3}^{\prime} \bar{z}_{3}^{\prime}+m_{2} w_{4} \bar{z}_{4}\right) \mathrm{d} x .
$$

They are Hilbert spaces because of the Dirichlet conditions imposed in (2.1). Note that the square of the induced norm

$$
\|w\|_{E}^{2}=\langle w, w\rangle_{E}=\frac{T_{1}}{2}\left\|w_{1}^{\prime}\right\|_{2}^{2}+\frac{m_{1}}{2}\left\|w_{2}\right\|_{2}^{2}+\frac{T_{2}}{2}\left\|w_{3}^{\prime}\right\|_{2}^{2}+\frac{m_{2}}{2}\left\|w_{4}\right\|_{2}^{2}
$$

is equal to the system's energy given by

$$
\mathcal{E}(t)=\frac{1}{2} \int_{-1}^{0}\left(m_{1}\left(\partial_{t} u\right)^{2}+T_{1}\left(\partial_{x} u\right)^{2}\right) \mathrm{d} x+\frac{1}{2} \int_{0}^{1}\left(m_{2}\left(\partial_{t} v\right)^{2}+T_{2}\left(\partial_{x} v\right)^{2}\right) \mathrm{d} x .
$$

For $\|\cdot\|_{E}$ we can check dissipativity of the generators below. The spaces $\mathcal{H}_{I I}$, $\mathcal{H}_{I I I}$, and $\mathcal{H}_{V I}$ are equipped with the modified scalar product

$$
\begin{aligned}
\left\langle\langle w, z\rangle_{E}=\right. & \frac{1}{2} \int_{-1}^{0}\left(T_{1} w_{1}^{\prime} \bar{z}_{1}^{\prime}+m_{1} w_{1} \bar{z}_{1}+m_{1} w_{2} \bar{z}_{2}\right) \mathrm{d} x \\
& +\frac{1}{2} \int_{0}^{1}\left(T_{2} w_{3}^{\prime} \bar{z}_{3}^{\prime}+m_{2} w_{3} \bar{z}_{3}+m_{2} w_{4} \bar{z}_{4}\right) \mathrm{d} x,
\end{aligned}
$$

whose induced norm is denoted by $\|\cdot\| \|_{E}$. Also these spaces are Hilbertian. For each $j \in J$, on $\mathcal{H}_{j}$ the norms $\|\cdot\|_{E}$ resp. $\|\cdot \cdot\|_{E}$, are equivalent to $\|\cdot\|_{\mathcal{H}}$.

In every case the generator corresponding to (1.1) is a restriction of the operator matrix

$$
\begin{aligned}
\mathcal{A} & =\left(\begin{array}{cccc}
0 & I & 0 & 0 \\
c_{1}^{2} D_{x x} & 0 & 0 & 0 \\
0 & 0 & 0 & I \\
0 & 0 & c_{2}^{2} D_{x x} & 0
\end{array}\right) \quad \text { with domain } \\
\mathcal{D} & =H^{2}(-1,0) \times H^{1}(-1,0) \times H^{2}(0,1) \times H^{1}(0,1)
\end{aligned}
$$

in $\mathcal{H}$. In view of the boundary and interface conditions in (1.8), we define

$$
\begin{gathered}
D\left(A_{I}\right)=\left\{w \in \mathcal{D} \cap \mathcal{H}_{I} \mid w_{2}(-1)=0=w_{4}(-1), T_{1} w_{1}^{\prime}(0)=T_{2} w_{3}^{\prime}(0),\right. \\
\left.w_{2}(0)-w_{4}(0)=-k T_{1} w_{1}^{\prime}(0)\right\}, \\
D\left(A_{I I}\right)=\left\{w \in \mathcal{D} \cap \mathcal{H}_{I I} \mid w_{2}(-1)=0, w_{3}^{\prime}(1)=0, T_{1} w_{1}^{\prime}(0)=T_{2} w_{3}^{\prime}(0),\right. \\
\left.w_{2}(0)-w_{4}(0)=-k T_{1} w_{1}^{\prime}(0)\right\}, \\
D\left(A_{I I I}\right)=\left\{w \in \mathcal{D} \mid w_{1}^{\prime}(-1)=0, w_{3}^{\prime}(1)=0, T_{1} w_{1}^{\prime}(0)=T_{2} w_{3}^{\prime}(0),\right. \\
\left.w_{2}(0)-w_{4}(0)=-k T_{1} w_{1}^{\prime}(0)\right\}, \\
D\left(A_{I V}\right)=\left\{w \in \mathcal{D} \cap \mathcal{H}_{I V} \mid w_{2}(-1)=0=w_{4}(1),\right. \\
\left.T_{1} w_{1}^{\prime}(0)-T_{2} w_{3}^{\prime}(0)=-k w_{2}(0)=-k w_{4}(0)\right\}, \\
D\left(A_{V}\right)=\left\{w \in \mathcal{D} \cap \mathcal{H}_{V} \mid w_{2}(-1)=0, w_{3}^{\prime}(1)=0,\right. \\
\left.T_{1} w_{1}^{\prime}(0)-T_{2} w_{3}^{\prime}(0)=-k w_{2}(0)=-k w_{4}(0)\right\},
\end{gathered}
$$




$$
\begin{aligned}
& D\left(A_{V I}\right)=\left\{w \in \mathcal{D} \cap \mathcal{H}_{V I} \mid\right. w_{1}^{\prime}(-1)=0=w_{3}^{\prime}(1), \\
&\left.T_{1} w_{1}^{\prime}(0)-T_{2} w_{3}^{\prime}(0)=-k w_{2}(0)=-k w_{4}(0)\right\},
\end{aligned}
$$

and set $A_{j} w=\mathcal{A} w$ for $w \in D\left(A_{j}\right)$. Some of the above equations for $w_{2}$ and $w_{4}$ are not explicitly stated in (1.8). They arise from differentiating the Dirichlet conditions on $w_{1}$ and $w_{3}$ in (1.8) with respect to time $t$, and are thus implicitly contained in the system. Observe that the restriction $A_{j}: D\left(A_{j}\right) \rightarrow \mathcal{H}_{j}$ actually maps into $\mathcal{H}_{j}$ for each $j \in J$.

We look for solutions $(u, v) \in C^{2}\left(\mathbb{R}_{+}, L^{2}(0,1)\right)^{2} \cap C\left(\mathbb{R}_{+}, H^{2}(0,1)\right)^{2}$ of the problem (1.1) with the boundary conditions given by (1.8). Existence and uniqueness of such solutions are established in Proposition 2.2 below.

This and most of the other results in this section are contained in [19] and [12] in a somewhat different form since there the wave equations (1.1) were rewritten as a first order system. However, the precise expression for the resolvent of $A_{j}$ is needed later on. Moreover, the transformation to the first order system has a one- or two-dimensional kernel in Cases II, III, and VI which was overlooked in [19] and [12]. In addition Case III was treated only partly there. We thus give most of the proofs within our framework.

We first show dissipativity and deal with the kernels of $A_{I I}, A_{I I I}$ and $A_{V I}$. For our main results we have to describe the spectral projection for the set $\{0\}$ of these operators, which will be achieved for $j=I I I$ only in Proposition 2.2. In the cases II and VI we argue as in [7].

Lemma 2.1. a) The operators $A_{j}$ are injective and dissipative on $\mathcal{H}_{j}$ for $j \in\{I, I V, V\}$.

b) Let $j \in\{I I, V I\}$. Then the operator $A_{j}-I$ is dissipative on $\mathcal{H}_{j}$. Moreover, $A_{j}$ has a one-dimensional kernel and a closed range with $\mathcal{H}_{j}=$ $\operatorname{ran} A_{j} \oplus \operatorname{ker} A_{j}$. The projections onto ker $A_{j}$ along $\operatorname{ran} A_{j}$ are given by $P_{I I} g=$ $\left(0,0, \phi_{I I}(g) \mathbb{1}, 0\right)$ and $P_{V I} g=\left(\phi_{V I}(g) \mathbb{1}, 0, \phi_{V I}(g) \mathbb{1}, 0\right)$ for the functionals $\phi_{j}$ defined in (2.5) respectively (2.7). On $\operatorname{ran} A_{j}$ the norm of $\mathcal{H}_{j}$ is equivalent to $\|\cdot\|_{E}$, and the part of $A_{j}$ in $\operatorname{ran} A_{j}$ is dissipative for $\langle\cdot, \cdot\rangle_{E}$.

c) The operator $A_{I I I}-I$ is dissipative on $\mathcal{H}_{I I I}$. The space of generalized eigenfunctions of $A_{I I I}$ for $\lambda=0$ is spanned by $(\mathbb{1}, 0,0,0),(0,0, \mathbb{1}, 0)$ and $(0, \mathbb{1}, 0, \mathbb{1})$, where the first two functions form a basis for $\operatorname{ker} A_{I I I}$.

Proof. 1) We first treat the cases $j \in\{I, I V, V\}$. Let $w \in D\left(A_{j}\right)$. Integrating by parts and using the boundary conditions at \pm 1 we compute

$$
\begin{aligned}
2\left\langle A_{j} w, w\right\rangle_{E}= & T_{1} \int_{-1}^{0}\left(w_{2}^{\prime} \bar{w}_{1}^{\prime}+w_{1}^{\prime \prime} \bar{w}_{2}\right) \mathrm{d} x+T_{2} \int_{0}^{1}\left(w_{4}^{\prime} \bar{w}_{3}^{\prime}+w_{3}^{\prime \prime} \bar{w}_{4}\right) \mathrm{d} x \\
= & T_{1} \int_{-1}^{0}\left(w_{2}^{\prime} \bar{w}_{1}^{\prime}-w_{1}^{\prime} \bar{w}_{2}^{\prime}\right) \mathrm{d} x+T_{2} \int_{0}^{1}\left(w_{4}^{\prime} \bar{w}_{3}^{\prime}-w_{3}^{\prime} \bar{w}_{4}^{\prime}\right) \mathrm{d} x \\
& +\left.T_{1} w_{1}^{\prime} \bar{w}_{2}\right|_{-1} ^{0}+\left.T_{2} w_{3}^{\prime} \bar{w}_{4}\right|_{0} ^{1}, \\
2 \operatorname{Re}\left\langle A_{j} w, w\right\rangle_{E}= & T_{1} w_{1}^{\prime}(0) \bar{w}_{2}(0)-T_{2} w_{3}^{\prime}(0) \bar{w}_{4}(0) .
\end{aligned}
$$

In case $\mathrm{I}$, the interface conditions in $D\left(A_{I}\right)$ then yield

$$
2 \operatorname{Re}\left\langle A_{I} w, w\right\rangle_{E}=-T_{1}^{2} k\left|w_{1}^{\prime}(0)\right|^{2} .
$$

Similarly, the conditions corresponding to (1.7) imply

$$
2 \operatorname{Re}\left\langle A_{j} w, w\right\rangle_{E}=-k\left|w_{2}(0)\right|^{2}
$$


for $j \in\{I V, V\}$. So these operators are dissipative. If $A_{j} w=0$, then the functions $w_{1}$ and $w_{3}$ are affine, whereas $w_{2}$ and $w_{4}$ vanish. The interface equations further yield $T_{1} w_{1}^{\prime}(0)=T_{2} w_{3}^{\prime}(0)$, and this number equals 0 if $j \in\{I, V\}$. Finally, the relations in $\mathcal{H}_{j}$ show that also $w_{1}=w_{3}=0$, and hence $A_{j}$ is injective for $j \in\{I, I V, V\}$.

2) We next look at the cases $j \in\{I I, I I I, V I\}$. Let $w \in D\left(A_{j}\right)$. As in (2.2)-(2.4) we compute

$$
\begin{aligned}
& 2 \operatorname{Re}\left\langle\left\langle A_{j} w, w\right\rangle_{E}\right. \\
& =T_{1} w_{1}^{\prime}(0) \bar{w}_{2}(0)-T_{2} w_{3}^{\prime}(0) \bar{w}_{4}(0)+m_{1} \operatorname{Re} \int_{-1}^{0} w_{2} \bar{w}_{1} \mathrm{~d} x+m_{2} \operatorname{Re} \int_{0}^{1} w_{4} \bar{w}_{3} \mathrm{~d} x \\
& \leq T_{1} w_{1}^{\prime}(0) \bar{w}_{2}(0)-T_{2} w_{3}^{\prime}(0) \bar{w}_{4}(0)+\frac{m_{1}}{2}\left(\left\|w_{1}\right\|_{2}^{2}+\left\|w_{2}\right\|_{2}^{2}\right)+\frac{m_{2}}{2}\left(\left\|w_{3}\right\|_{2}^{2}+\left\|w_{4}\right\|_{2}^{2}\right) \\
& \leq \frac{m_{1}}{2}\left(\left\|w_{1}\right\|_{2}^{2}+\left\|w_{2}\right\|_{2}^{2}\right)+\frac{m_{2}}{2}\left(\left\|w_{3}\right\|_{2}^{2}+\left\|w_{4}\right\|_{2}^{2}\right) .
\end{aligned}
$$

Consequently, $A_{j}-I$ is dissipative.

Let $A_{j} w=0$. As above, then $w_{1}$ and $w_{3}$ are affine, whereas $w_{2}$ and $w_{4}$ are zero. The Neumann conditions in the domains imply that $w_{1}$ and $w_{3}$ are constant. The Dirichlet conditions in $\mathcal{H}_{j}$ now yield that $\operatorname{ker} A_{I I}$ is spanned by $(0,0, \mathbb{1}, 0)$ and ker $A_{V I}$ by $(\mathbb{1}, 0, \mathbb{1}, 0)$, whereas ker $A_{I I I}$ has the basis $\{(\mathbb{1}, 0,0,0),(0,0, \mathbb{1}, 0)\}$.

Morever, a function $w \in D\left(A_{I I I}\right)$ satisfies $A_{I I I} w=(a \mathbb{1}, 0, b \mathbb{1}, 0)$ for some numbers $a, b \in \mathbb{C}$ if and only if all components $w_{j}$ are constant and $w_{2}=a \mathbb{1}$ is equal to $w_{4}=b \mathbb{1}$.

3) To establish the asserted decompositions in b) for $j \in\{I I, V I\}$, we first introduce the bounded linear map

$$
\phi_{I I}: \mathcal{H}_{I I} \rightarrow \mathbb{C} ; \quad \phi_{I I}\left(g_{1}, g_{2}, g_{3}, g_{4}\right)=g_{3}(0)-g_{1}(0)+\frac{k T_{2}}{c_{2}^{2}} \int_{0}^{1} g_{4}(x) \mathrm{d} x .
$$

First, let $f=A_{I I} w$ belong to the range of $A_{I I}$. We then deduce the equations $w_{2}=f_{1}, w_{4}=f_{3}$,

$$
\begin{aligned}
& w_{1}(x)=a(1+x)+\frac{1}{c_{1}^{2}} \int_{-1}^{x}(x-s) f_{2}(s) \mathrm{d} s, \quad x \in[-1,0], \\
& w_{3}(x)=b+\frac{1}{c_{2}^{2}} \int_{x}^{1}(s-x) f_{4}(s) \mathrm{d} s, \quad x \in[0,1],
\end{aligned}
$$

for complex numbers $a$ and $b$, taking into account the boundary conditions $w_{1}(-1)=0$ and $w_{3}^{\prime}(1)=0$. Note that

$$
w_{1}^{\prime}(0)=a+\frac{1}{c_{1}^{2}} \int_{-1}^{0} f_{2}(s) \mathrm{d} s \quad \text { and } \quad w_{3}^{\prime}(0)=-\frac{1}{c_{2}^{2}} \int_{0}^{1} f_{4}(s) \mathrm{d} s .
$$

The interface conditions in $D\left(A_{I I}\right)$ then determine the parameter $a$ and imply that the vector $f$ belongs to $\operatorname{ker} \phi_{I I}$.

Conversely, let $f \in \mathcal{H}_{I I}$ be contained in the kernel of $\phi_{I I}$. We then define $w$ by $w_{2}=f_{1}, w_{4}=f_{3}$, and (2.6) with $b=0$ and

$$
a=\frac{f_{3}(0)-f_{1}(0)}{k T_{1}}-\frac{1}{c_{1}^{2}} \int_{-1}^{0} f_{2}(x) \mathrm{d} x=-\frac{T_{2}}{T_{1} c_{2}^{2}} \int_{0}^{1} f_{4}(x) \mathrm{d} x-\frac{1}{c_{1}^{2}} \int_{-1}^{0} f_{2}(x) \mathrm{d} x,
$$


where we have used $\phi_{I I}(f)=0$ in the second equation. One can now check that $w$ belongs to $D\left(A_{I I}\right)$, and we have $A_{I I} w=f$ by construction. Therefore, $\operatorname{ran} A_{I I}=\operatorname{ker} \phi_{I I}$ is closed and the map $P_{I I} g=\left(0,0, \phi_{I I}(g) \mathbb{1}, 0\right)$ is the bounded projection onto ker $A_{I I}$ along ran $A_{I I}$.

Let $g \in \operatorname{ran} A_{I I}$. We clearly have $\|g\|_{E}^{2} \leq\|g\| \|$ and also $\left\|g_{1}\right\|_{H^{1}} \leq c\left\|g_{1}^{\prime}\right\|_{L^{2}}$ since $g_{1}(-1)=0$, for some constants $c>0$. Due to $\phi_{I I}(g)=0$, we can then bound $\left|g_{3}(0)\right|$ by $c\left(\left\|g_{1}^{\prime}\right\|_{L^{2}}+\left\|g_{4}\right\|_{L^{2}}\right)$. Hence, the norm $\|\mid \cdot\|$ is equivalent to $\|\cdot\|_{E}$ on $\operatorname{ran} A_{I I}$. The dissipativity of the part of $A_{I I}$ in $\operatorname{ran} A_{I I}$ is proved as in (2.3).

The remaining assertions for $A_{V I}$ are similarly shown using the functional

$$
\phi_{V I}: \mathcal{H}_{V I} \rightarrow \mathbb{C} ; \quad \phi_{V I}(f)=\frac{T_{1}}{k c_{1}^{2}} \int_{-1}^{0} f_{2}(s) \mathrm{d} s+\frac{T_{2}}{k c_{2}^{2}} \int_{0}^{1} f_{4}(s) \mathrm{d} s+f_{1}(0)
$$

and the projection $P_{V I} g=\left(\phi_{V I}(g) \mathbb{1}, 0, \phi_{V I}(g) \mathbb{1}, 0\right)$.

We next describe the spectrum of $A_{j}$ and compute its resolvent. Take $j \in J, f \in \mathcal{H}_{j}$, and $\lambda \in \mathbb{C} \backslash\{0\}$. We introduce the numbers

$$
a_{l}=\frac{T_{l}}{c_{l}}, \quad \omega_{l}=\frac{\lambda}{c_{l}}, \quad c_{l}=\frac{\sqrt{T_{l}}}{\sqrt{m_{l}}}, \quad d=\frac{c_{2}}{c_{1}}
$$

for $\lambda \in \mathbb{C}$ and $l \in\{1,2\}$, and the functions

$$
\begin{aligned}
g_{j}: \mathbb{C} \rightarrow \mathbb{C} ; & g_{j}(z)= \begin{cases}\sinh \left(z+\omega_{1}\right), & j \in\{I, I I, I V, V\}, \\
\cosh \left(z+\omega_{1}\right), & j \in\{I I I, V I\},\end{cases} \\
h_{j}: \mathbb{C} \rightarrow \mathbb{C} ; & h_{j}(z)= \begin{cases}\sinh \left(\omega_{2}-z\right), & j \in\{I, I V\}, \\
\cosh \left(\omega_{2}-z\right), & j \in\{I I, I I I, V, V I\} .\end{cases}
\end{aligned}
$$

The function sinh corresponds to Dirichlet conditions at $x=-1$ for $g_{j}$ and at $x=1$ for $h_{j}$, whereas cosh is used in the Neumann cases. We further set

$$
\begin{aligned}
& U_{\lambda}(x)=\frac{-1}{c_{1} \lambda} \int_{-1}^{x} \sinh \left(\omega_{1}(x-r)\right)\left(\lambda f_{1}(r)+f_{2}(r)\right) \mathrm{d} r, \quad x \in[-1,0], \\
& W_{\lambda}(x)=\frac{-1}{c_{2} \lambda} \int_{x}^{1} \sinh \left(\omega_{2}(r-x)\right)\left(\lambda f_{3}(r)+f_{4}(r)\right) \mathrm{d} r, \quad x \in[0,1] .
\end{aligned}
$$

All solutions $w \in \mathcal{D}$ to $\lambda w-\mathcal{A} w=f$ satisfying the boundary conditions at $x= \pm 1$ for Case $j$ in (1.8) are given by

$$
\begin{array}{lll}
w_{1}=\alpha(\lambda) g_{j}\left(\omega_{1} \cdot\right)+U_{\lambda}, & w_{2}=\lambda w_{1}-f_{1} & \text { on }[-1,0], \\
w_{3}=\beta(\lambda) h_{j}\left(\omega_{2} \cdot\right)+W_{\lambda}, & w_{4}=\lambda w_{3}-f_{3} & \text { on }[0,1],
\end{array}
$$

for some numbers $a(\lambda), b(\lambda) \in \mathbb{C}$. Since $f$ belongs to $\mathcal{H}_{j}$, these functions $w_{2}$ and $w_{4}$ satisfy the Dirichlet conditions at $x= \pm 1$ in $D\left(A_{j}\right)$.

We next impose the interface conditions induced by (1.6) for $j \in\{I, I I, I I I\}$ and by (1.7) for $j \in\{I V, V, V I\}$. They are satisfied if and only if the solution $w$ fulfill the equations

$$
\begin{aligned}
\lambda a_{1} \alpha(\lambda) g_{j}^{\prime}(0)+T_{1} U_{\lambda}^{\prime}(0) & =\lambda a_{2} \beta(\lambda) h_{j}^{\prime}(0)+T_{2} W_{\lambda}^{\prime}(0), \\
\lambda \alpha(\lambda) g_{j}(0)+\lambda U_{\lambda}(0)-f_{1}(0)-\lambda \beta(\lambda) h_{j}(0)-\lambda W_{\lambda}(0)+f_{3}(0) & \left.=-k\left(\lambda a_{1} \alpha(\lambda) g_{j}^{\prime}(0)+T_{1} U_{\lambda}^{\prime}(0)\right)\right) .
\end{aligned}
$$


if $j \in\{I, I I, I I I\}$, as well as

$$
\begin{aligned}
& \lambda \alpha(\lambda) g_{j}(0)+\lambda U_{\lambda}(0)=\lambda \beta(\lambda) h_{j}(0)+\lambda W_{\lambda}(0), \\
& \begin{array}{r}
\lambda a_{1} \alpha(\lambda) g_{j}^{\prime}(0)+T_{1} U_{\lambda}^{\prime}(0)-\lambda a_{2} \beta(\lambda) h_{j}^{\prime}(0)-T_{2} W_{\lambda}^{\prime}(0) \\
=-k\left(\lambda \alpha(\lambda) g_{j}(0)+\lambda U_{\lambda}(0)-f_{1}(0)\right) .
\end{array}
\end{aligned}
$$

if $j \in\{I V, V, V I\}$. (For $w_{2}$ and $w_{4}$ we employ (2.9) and $f \in \mathcal{H}_{j}$.)

We thus obtain a solution $w \in D\left(A_{j}\right)$ to $\lambda w-\mathcal{A} w=f$ if and only if the coefficients $(a(\lambda), b(\lambda))$ solve the system

$$
\begin{aligned}
M_{j}(\lambda)\left(\begin{array}{c}
\alpha(\lambda) \\
\beta(\lambda)
\end{array}\right) & =\left(\begin{array}{c}
-T_{1} U_{\lambda}^{\prime}(0)+T_{2} W_{\lambda}^{\prime}(0) \\
-\lambda U_{\lambda}(0)+\lambda W_{\lambda}(0)-k T_{1} U_{\lambda}^{\prime}(0)+f_{1}(0)-f_{3}(0)
\end{array}\right), \\
M_{j}(\lambda) & =\lambda\left(\begin{array}{cc}
a_{1} g_{j}^{\prime}(0) & -a_{2} h_{j}^{\prime}(0) \\
g_{j}(0)+a_{1} k g_{j}^{\prime}(0) & -h_{j}(0)
\end{array}\right)
\end{aligned}
$$

in the cases $j \in\{I, I I, I I I\}$, respectively

$$
\begin{aligned}
M_{j}(\lambda)\left(\begin{array}{c}
\alpha(\lambda) \\
\beta(\lambda)
\end{array}\right) & =\left(\begin{array}{c}
-\lambda U_{\lambda}(0)+\lambda W_{\lambda}(0) \\
-T_{1} U_{\lambda}^{\prime}(0)+T_{2} W_{\lambda}^{\prime}(0)-k \lambda U_{\lambda}(0)+k f_{1}(0)
\end{array}\right), \\
M_{j}(\lambda) & =\lambda\left(\begin{array}{cc}
g_{j}(0) & -h_{j}(0) \\
a_{1} g_{j}^{\prime}(0)+k g_{j}(0) & -a_{2} h_{j}^{\prime}(0)
\end{array}\right)
\end{aligned}
$$

for $j \in\{I V, V, V I\}$. The determinant of $M_{j}(\lambda)$ is given by

$$
\begin{aligned}
\operatorname{det} M_{j}(\lambda) & =-\lambda^{2} \Delta_{j}(\lambda), \quad j \in\{I, I I, I I I\}, \\
\operatorname{det} M_{j}(\lambda) & =\lambda^{2} \Delta_{j}(\lambda), \quad j \in\{I V, V, V I\}, \\
\Delta_{I}(\lambda) & =\left[\sinh \left(\omega_{1}\right)+a_{1} k \cosh \left(\omega_{1}\right)\right] a_{2} \cosh \left(\omega_{2}\right)+a_{1} \cosh \left(\omega_{1}\right) \sinh \left(\omega_{2}\right), \\
\Delta_{I I}(\lambda) & =\left[\sinh \left(\omega_{1}\right)+a_{1} k \cosh \left(\omega_{1}\right)\right] a_{2} \sinh \left(\omega_{2}\right)+a_{1} \cosh \left(\omega_{1}\right) \cosh \left(\omega_{2}\right), \\
\Delta_{I I I}(\lambda) & =\left[\cosh \left(\omega_{1}\right)+a_{1} k \sinh \left(\omega_{1}\right)\right] a_{2} \sinh \left(\omega_{2}\right)+a_{1} \sinh \left(\omega_{1}\right) \cosh \left(\omega_{2}\right), \\
\Delta_{I V}(\lambda) & =\left[a_{1} \cosh \left(\omega_{1}\right)+k \sinh \left(\omega_{1}\right)\right] \sinh \left(\omega_{2}\right)+a_{2} \sinh \left(\omega_{1}\right) \cosh \left(\omega_{2}\right), \\
\Delta_{V}(\lambda) & =\left[a_{1} \cosh \left(\omega_{1}\right)+k \sinh \left(\omega_{1}\right)\right] \cosh \left(\omega_{2}\right)+a_{2} \sinh \left(\omega_{1}\right) \sinh \left(\omega_{2}\right), \\
\Delta_{V I}(\lambda) & =\left[a_{1} \sinh \left(\omega_{1}\right)+k \cosh \left(\omega_{1}\right)\right] \cosh \left(\omega_{2}\right)+a_{2} \cosh \left(\omega_{1}\right) \sinh \left(\omega_{2}\right) .
\end{aligned}
$$

If $\Delta_{j}(\lambda)=0$ for some $\lambda \neq 0$, the above observations yield a non-zero solution $w \in D\left(A_{j}\right)$ of $\lambda w-A_{j} w=0$, and thus $\lambda$ is an eigenvalue of $A_{j}$. Otherwise we have constructed a unique solution $w \in D\left(A_{j}\right)$ of the equation $\lambda w-A_{j} w=f$ for the given function $f \in \mathcal{H}_{j}$, where the map $f \mapsto w$ is continuous in $\mathcal{H}_{j}$. Hence, $\lambda \neq 0$ belongs to the resolvent set if $\Delta_{j}(\lambda) \neq 0$.

Observe that the functions $\Delta_{j}$ are positive on $(0, \infty)$, so that the resolvent of $A_{j}$ exists for $\lambda>0$, and that it is compact. Because of these properties and Lemma 2.1, $A_{j}$ is invertible for $j \in\{I, I V, V\}$ and 0 is an isolated eigenvalue of $A_{j}$ for $j \in\{I I, I I I, V I\}$.

We state these facts and several consequences in the next basic proposition, using the following notation. The spectrum of a closed operator $B$ is denoted by $\sigma(A)$ and its point spectrum by $\sigma_{p}(B)$. We write $\mathbb{C}_{-}=\{\lambda \epsilon$ $\mathbb{C} \mid \operatorname{Re} \lambda<0\}$.

Proposition 2.2. a) The resolvent of $A_{j}$ is compact, and we have

$$
\sigma\left(A_{j}\right)=\sigma_{p}\left(A_{j}\right)=\left\{\lambda \in \mathbb{C} \backslash\{0\} \mid \Delta_{j}(\lambda)=0\right\}, \quad j \in\{I, I V, V\},
$$




$$
\sigma\left(A_{j}\right)=\sigma_{p}\left(A_{j}\right)=\{0\} \cup\left\{\lambda \in \mathbb{C} \backslash\{0\} \mid \Delta_{j}(\lambda)=0\right\}, \quad j \in\{I I, I I I, V I\} .
$$

b) For $j \in\{I, I V, V\}$ the operator $A_{j}$ generates a contractive $C_{0}$-semigroup $T_{j}(\cdot)$ on $\mathcal{H}_{j}$.

c) Let $j \in\{I I, I I I, V I\}$. Then 0 is an isolated eigenvalue of $A_{j}$. Let $P_{j}$ be the corresponding spectral projection and $A_{j}^{1}$ be the restriction of $A_{j}$ to $D\left(A_{j}\right) \cap \operatorname{ker} P_{j}$. Then $P_{I I}$ and $P_{V I}$ are given as in Lemma 2.1 and $P_{I I I}$ by (2.14). We have $\sigma\left(A_{j}^{1}\right)=\sigma\left(A_{j}\right) \backslash\{0\}$.

For $j \in\{I I, I V\}$ the operator $A_{j}$ generates a bounded $C_{0}$-semigroup $T_{j}(\cdot)$ on $\mathcal{H}_{j}$. The restriction of $T_{j}(\cdot)$ to $\operatorname{ran} P_{j}=\operatorname{ker} A_{j}$ is constant and that to $\operatorname{ker} P_{j}=\operatorname{ran} A_{j}$ is contractive for $\|\cdot\|_{E}$ and has the generator $A_{j}^{1}$.

On ker $P_{I I I}$ the norm of $\mathcal{H}_{I I I}$ is equivalent to $\|\cdot\|_{E}$. The operator $A_{I I I}$ generates a $C_{0}$-semigroup $T_{I I I}(\cdot)$ on $\mathcal{H}_{I I I}$ with linear growth. The restriction of $T_{I I I}(\cdot)$ to $\operatorname{ran} P_{j}$ is affine in $t$ and that to $\operatorname{ker} P_{j}$ is contractive for $\|\cdot\|_{E}$ and has the generator $A_{I I I}^{1}$.

c) In each case, for every $w_{0}=\left(u_{0}, u_{1}, v_{0}, v_{1}\right) \in D\left(A_{j}\right)$ the unique solution $(u, v)$ of (1.1) with the boundary conditions of (1.8) is given by $w(t)=$ $\left(u(t), \partial_{t} u(t), v(t), \partial_{t} v(t)\right)=T_{j}(t) w_{0}$ for $t \geq 0$.

d) Let $d=c_{2} / c_{1} \notin \mathbb{Q}$. Then $\sigma\left(A_{j}\right) \subseteq \mathbb{C}_{-}$for $j \in\{I, I V, V\}$ and $\sigma\left(A_{j}^{1}\right) \subseteq \mathbb{C}_{-}$ for $j \in\{I I, I I I, V I\}$. The resolvent is bounded by

$$
\left\|R\left(i s, A_{j}\right)\right\| \leq \frac{c}{\left|\Delta_{j}(i s)\right|}
$$

for a constant $c>0$, all $s \in \mathbb{R}$ with $|s| \geq 1$, and $j \in J$.

Proof. 1) Assertion a) was shown above, and we have seen that $(0, \infty)$ belongs to the resolvent set for each $j \in J$. Hence, Lemma 2.1 and the LumerPhillips theorem show that $A_{j}$ generates a $C_{0}$-semigroup on $\mathcal{H}_{j}$ which is contractive for $j \in\{I, I V, V\}$. Part c) is deduced from the generation property by a standard calculation.

Let $j \in\{I I, V I\}$. The operator $A_{j}$ has no proper generalized eigenvector for $\lambda=0$ since $\operatorname{ker} A_{j} \cap \operatorname{ran} A_{j}=\{0\}$. Moreover, the projections $P_{j}$ from Lemma 2.1 map onto ker $A_{j}$ and commute with $A_{j}$. They thus coincide with the spectral projection of $A_{j}$ for $\{0\}$ which implies the assertion $\sigma\left(A_{j}^{1}\right)=$ $\sigma\left(A_{j}\right) \backslash\{0\}$. Clearly, $T_{j}(\cdot)$ leaves invariant the decomposition $\mathcal{H}_{j}=\operatorname{ran} A_{j} \oplus$ $\operatorname{ker} A_{j}$ and its restrictions to $\operatorname{ran} A_{j}$ and ker $A_{j}$ are generated by $A_{j}^{1}$ and 0 , respectively. Since $A_{j}^{1}$ is dissipative for the equivalent norm $\|\cdot\|_{E}$ on $\operatorname{ran} A_{j}$ by Lemma 2.1, the semigroup $T_{j}(\cdot)$ is bounded on $\operatorname{ran} A_{j}$ and hence on $\mathcal{H}_{j}$.

2 ) We next prove part c) also for $j=I I I$. Take a number $\epsilon>0$ such that $\Delta_{I I I}$ is non-zero on $B(0,2 \epsilon) \backslash\{0\}$. The spectral projection $P_{I I I}$ is given by

$$
P_{I I I} f=\frac{1}{2 \pi i} \int_{|\lambda|=\epsilon} R\left(\lambda, A_{I I I}\right) f \mathrm{~d} \lambda=\frac{1}{2 \pi i} \int_{|\lambda|=\epsilon}\left(\begin{array}{c}
\alpha(\lambda) \cosh \left(\lambda(\cdot+1) / c_{1}\right) \\
\lambda \alpha(\lambda) \cosh \left(\lambda(\cdot+1) / c_{1}\right) \\
\beta(\lambda) \cosh \left(\lambda(\cdot-1) / c_{2}\right) \\
\lambda \beta(\lambda) \cosh \left(\lambda(\cdot-1) / c_{2}\right)
\end{array}\right) \mathrm{d} \lambda .
$$

for $f \in \mathcal{H}_{I I I}$, where we use (2.8)-(2.10) and Cauchy's integral theorem and formula. (Observe that the integrands of $U_{\lambda}$ and $W_{\lambda}$ vanish at $\lambda=0$.) 
Equation (2.10) next yields

$$
\left(\begin{array}{l}
\alpha(\lambda) \\
\beta(\lambda)
\end{array}\right)=\frac{1}{\lambda^{2}} \varphi(\lambda) z(\lambda)
$$

where the factors are given by $\varphi(\lambda)=\lambda / \Delta_{I I I}(\lambda)$ for $\lambda \epsilon B(0,2 \epsilon) \backslash\{0\}$ and

$$
\begin{aligned}
& z_{1}(\lambda)=\cosh \left(\lambda / c_{2}\right)\left(-T_{1} U_{\lambda}^{\prime}(0)+T_{2} W_{\lambda}^{\prime}(0)\right)+a_{2} \sinh \left(\lambda / c_{2}\right)\left(-\lambda U_{\lambda}(0)\right. \\
& \left.+\lambda W_{\lambda}(0)-k T_{1} U_{\lambda}^{\prime}(0)+f_{1}(0)-f_{3}(0)\right) \\
& z_{2}(\lambda)=\left(\cosh \left(\lambda / c_{1}\right)+a_{1} k \sinh \left(\lambda / c_{1}\right)\right)\left(-T_{1} U_{\lambda}^{\prime}(0)+T_{2} W_{\lambda}^{\prime}(0)\right) \\
& -a_{1} \sinh \left(\lambda / c_{1}\right)\left(-\lambda U_{\lambda}(0)+\lambda W_{\lambda}(0)-k T_{1} U_{\lambda}^{\prime}(0)+f_{1}(0)-f_{3}(0)\right)
\end{aligned}
$$

for $\lambda \in \mathbb{C}$. Note that $z$ is holomorphic on $\mathbb{C}$. The power series of sinh and cosh imply the expansion

$\Delta_{I I I}(\lambda)=\left(\frac{a_{1}}{c_{1}}+\frac{a_{2}}{c_{2}}\right) \lambda+\frac{a_{1} a_{2} k}{c_{1} c_{2}} \lambda^{2}+\mathcal{O}\left(\lambda^{3}\right)=\left(m_{1}+m_{2}\right) \lambda+k m_{1} m_{2} \lambda^{2}+\mathcal{O}\left(\lambda^{3}\right)$

near $\lambda=0$, so that $\varphi$ has a holomorphic extension at 0 with $\varphi(0)=\frac{1}{m_{1}+m_{2}}$. Moreover, its derivative is given by

$$
\begin{aligned}
\varphi^{\prime}(\lambda) & =\frac{\Delta_{I I I}(\lambda)-\lambda \Delta_{I I I}^{\prime}(\lambda)}{\Delta_{I I I}(\lambda)^{2}}=\frac{-k m_{1} m_{2} \lambda^{2}+\mathcal{O}\left(\lambda^{3}\right)}{\left(m_{1}+m_{2}\right)^{2} \lambda^{2}+\mathcal{O}\left(\lambda^{3}\right)}, \\
\varphi^{\prime}(0) & =\frac{-k m_{1} m_{2}}{\left(m_{1}+m_{2}\right)^{2}} .
\end{aligned}
$$

By means of Cauchy's integral formula, we can now evaluate the above expression for $P_{I I I} f$ and derive

$$
\begin{aligned}
P_{I I I} f & =\left(\frac{\mathrm{d}}{\mathrm{d} \lambda}\right)_{\mid \lambda=0}\left(\begin{array}{c}
\varphi(\lambda) z_{1}(\lambda) \cosh \left(\lambda(\cdot+1) / c_{1}\right) \\
\lambda \varphi(\lambda) z_{1}(\lambda) \cosh \left(\lambda(\cdot+1) / c_{1}\right) \\
\varphi(\lambda) z_{2}(\lambda) \cosh \left(\lambda(\cdot-1) / c_{2}\right) \\
\lambda \varphi(\lambda) z_{2}(\lambda) \cosh \left(\lambda(\cdot-1) / c_{2}\right)
\end{array}\right) \\
& =\frac{-k m_{1} m_{2}}{\left(m_{1}+m_{2}\right)^{2}}\left(\begin{array}{c}
z_{1}(0) \mathbb{1} \\
0 \\
z_{2}(0) \mathbb{1} \\
0
\end{array}\right)+\frac{1}{m_{1}+m_{2}}\left(\begin{array}{c}
0 \\
z_{1}(0) \mathbb{1} \\
0 \\
z_{2}(0) \mathbb{1}
\end{array}\right)+\frac{1}{m_{1}+m_{2}}\left(\begin{array}{c}
z_{1}^{\prime}(0) \mathbb{1} \\
0 \\
z_{2}^{\prime}(0) \mathbb{1} \\
0
\end{array}\right) .
\end{aligned}
$$

To compute the vectors $z(0)$ and $z^{\prime}(0)$, we first infer from (2.8) the identities

$$
\begin{aligned}
U_{\lambda}^{\prime}(0) & =-\frac{1}{c_{1}^{2}} \int_{-1}^{0} \cosh \left(\lambda r / c_{1}\right)\left(\lambda f_{1}(r)+f_{2}(r)\right) \mathrm{d} r \\
W_{\lambda}^{\prime}(0) & =\frac{1}{c_{2}^{2}} \int_{0}^{1} \cosh \left(\lambda r / c_{1}\right)\left(\lambda f_{3}(r)+f_{4}(r)\right) \mathrm{d} r
\end{aligned}
$$

and hence

$$
z(0)=\left(\begin{array}{l}
\frac{T_{1}}{c_{1}^{2}} \int_{-1}^{0} f_{2} \mathrm{~d} r+\frac{T_{2}}{c_{2}^{2}} \int_{0}^{1} f_{4} \mathrm{~d} r \\
\frac{T_{1}}{c_{1}^{2}} \int_{-1}^{0} f_{2} \mathrm{~d} r+\frac{T_{2}}{c_{2}^{2}} \int_{0}^{1} f_{4} \mathrm{~d} r
\end{array}\right)=\left(\begin{array}{l}
m_{1} \int_{-1}^{0} f_{2} \mathrm{~d} r+m_{2} \int_{0}^{1} f_{4} \mathrm{~d} r \\
m_{1} \int_{-1}^{0} f_{2} \mathrm{~d} r+m_{2} \int_{0}^{1} f_{4} \mathrm{~d} r
\end{array}\right) .
$$

We further compute

$$
\frac{\mathrm{d}}{\mathrm{d} \lambda} U_{\lambda}^{\prime}(0)=-\frac{1}{c_{1}^{2}} \int_{-1}^{0}\left(\frac{1}{c_{1}} \sinh \left(\frac{\lambda r}{c_{1}}\right)\left(\lambda f_{1}(r)+f_{2}(r)\right)+\cosh \left(\frac{\lambda r}{c_{1}}\right) f_{1}(r)\right) \mathrm{d} r,
$$




$$
\begin{aligned}
\frac{\mathrm{d}}{\mathrm{d} \lambda} U_{\lambda}^{\prime}(0)_{\mid \lambda=0} & =-\frac{1}{c_{1}^{2}} \int_{-1}^{0} f_{1} \mathrm{~d} r, \\
\frac{\mathrm{d}}{\mathrm{d} \lambda} W_{\lambda}^{\prime}(0)_{\mid \lambda=0} & =\frac{1}{c_{2}^{2}} \int_{0}^{1} f_{3} \mathrm{~d} r .
\end{aligned}
$$

As a result,

$z_{1}^{\prime}(0)=m_{1} \int_{-1}^{0} f_{1} \mathrm{~d} r+m_{2} \int_{0}^{1} f_{3} \mathrm{~d} r+k m_{1} m_{2} \int_{-1}^{0} f_{2} \mathrm{~d} r+m_{2} f_{1}(0)-m_{2} f_{3}(0)$,
$z_{2}^{\prime}(0)=m_{1} \int_{-1}^{0} f_{1} \mathrm{~d} r+m_{2} \int_{0}^{1} f_{3} \mathrm{~d} r+k m_{1} m_{2} \int_{0}^{1} f_{4} \mathrm{~d} r-m_{1} f_{1}(0)+m_{1} f_{3}(0)$.

Therefore a function $f \in \mathcal{H}_{I I I}$ belongs to ker $P_{I I I}$ if and only if $0=z_{1}(0)=$ $z_{1}^{\prime}(0)=z_{2}^{\prime}(0)$; i.e.,

$$
\begin{array}{r}
m_{1} \int_{-1}^{0} f_{2} \mathrm{~d} r+m_{2} \int_{0}^{1} f_{4} \mathrm{~d} r=0, \\
\frac{m_{1}+m_{2}}{m_{2}} \int_{-1}^{0} f_{1} \mathrm{~d} r+\frac{m_{1}+m_{2}}{m_{1}} \int_{0}^{1} f_{3} \mathrm{~d} r=0, \\
f_{1}(0)-f_{3}(0)+k m_{1} \int_{-1}^{0} f_{2} \mathrm{~d} r=0 .
\end{array}
$$

We can now show that the norm $\|\cdot\|_{E}$ is equivalent to $\|\cdot\| \|_{E}$ on ker $P_{I I I}$. Let $f \in \operatorname{ker} P_{I I I}$. We use the equations

$$
f_{l}(x)=f_{l}(0)+\int_{0}^{x} f_{l}^{\prime}(r) \mathrm{d} r
$$

for $l \in\{1,3\}$ and $x \in[-1,0]$, resp. $x \in[0,1]$. Formulas $(2.16)$ then yield

$$
\begin{aligned}
& \frac{m_{1}+m_{2}}{m_{2}} f_{1}(0)+\frac{m_{1}+m_{2}}{m_{1}} f_{3}(0)=-\frac{m_{1}+m_{2}}{m_{2}} \int_{-1}^{0} \int_{0}^{x} f_{1}^{\prime}(r) \mathrm{d} r \mathrm{~d} x \\
&-\frac{m_{1}+m_{2}}{m_{1}} \int_{0}^{1} \int_{0}^{x} f_{3}^{\prime}(r) \mathrm{d} r \mathrm{~d} x, \\
& f_{1}(0)-f_{3}(0)=-k m_{1} \int_{-1}^{0} f_{2} \mathrm{~d} r .
\end{aligned}
$$

Here the coeffcient matrix in front of the vector $\left(f_{1}(0), f_{3}(0)\right)$ is invertible. We can thus bound $\left|f_{1}(0)\right|$ and $\left|f_{3}(0)\right|$ by $c\|f\|_{\mathcal{H}}$ for a constant $c>0$. The claimed equivalence then easily follows from (2.17).

As in $(2.3)$ we see that $A_{I I I}$ is dissipative for $\|\cdot\|_{E}$. Its restriction $A_{I I I}^{1}$ to ker $P_{I I I}$ thus generates a bounded semigroup that coincides with the restriction of $T_{I I I}(\cdot)$ to ker $P_{I I I}$. Moreover, for $w_{0} \in \operatorname{ran} P_{I I I}$ the solution $T_{I I I}(t) w_{0}$ is a linear combination of functions $(\mathbb{1}, 0,0,0),(0,0, \mathbb{1}, 0)$, and $t(0, \mathbb{1}, 0, \mathbb{1})$ by Lemma 2.1. Altogether we have shown assertion $\mathrm{c})$.

3) We now look at the purely imaginary non-zero eigenvalues of $A_{I I I}$. By part a), is $\in \mathbb{R} \backslash\{0\}$ is an eigenvalue if and only if

$$
\begin{aligned}
0=\Delta_{I I I}(i s)=- & a_{1} a_{2} k \sin \left(\frac{s}{c_{1}}\right) \sin \left(\frac{s}{c_{2}}\right) \\
& +i\left[a_{2} \cos \left(\frac{s}{c_{1}}\right) \sin \left(\frac{s}{c_{2}}\right)+a_{1} \sin \left(\frac{s}{c_{1}}\right) \cos \left(\frac{s}{c_{2}}\right)\right] .
\end{aligned}
$$

Hence, $s / c_{1}$ or $s / c_{2}$ have to be a zero of $\sin$. In the first case, the second summand of $\operatorname{Im} \Delta_{I I I}(i s)$ vanishes and $\cos \left(s / c_{1}\right)$ has absolute value one. As a result, also $\sin \left(s / c_{2}\right)$ has to be zero. Here we can interchange the role of $s / c_{1}$ and $s / c_{2}$, so that both numbers must belong to $\pi \mathbb{Z}$. This means that 
is $\in \mathbb{R} \backslash\{0\}$ is an eigenvalue of $A_{I I I}$ if and only if $s=c_{1} k \pi=c_{2} l \pi$ for some integers $k$ and $l$, which is equivalent to $d \in \mathbb{Q}$.

4) Let $d$ be irrational. The spectral assertions in d) follow from part a) as well as formula (4.4) of [19], Theorem 1 of [12] for $j=I V$, and step 3) for $j=I I I$. Let $f \in \mathcal{H}_{j}, s \in \mathbb{R} \backslash\{0\}$, and $w=R\left(i s, A_{j}\right) f$. Observe that $\sinh (i s)=i \sin s$ and $\cosh (i s)=\cos s$. We first take $j \in\{I, I V, V\}$. Formulas (2.9)-(2.12) and (2.15) with $\lambda=i s$ imply the inequalities

$$
\begin{aligned}
\|w\|_{E} \leq & c\left(\left\|w_{1}^{\prime}\right\|_{L_{2}}+\left\|w_{2}\right\|_{L_{2}}+\left\|w_{3}^{\prime}\right\|_{L_{2}}+\left\|w_{4}\right\|_{L_{2}}\right) \\
\leq & c\left(|s \alpha(i s)|+\left\|U_{i s}^{\prime}\right\|_{L^{2}}+\left\|s U_{i s}\right\|_{L^{2}}+\left\|f_{1}\right\|_{L^{2}}+|s \beta(i s)|+\left\|W_{i s}^{\prime}\right\|_{L^{2}}\right. \\
& \left.\quad+\left\|s W_{i s}\right\|_{L^{2}}+\left\|f_{3}\right\|_{L^{2}}\right) \\
\leq & \frac{c}{|\Delta(i s)|}\left(\left\|U_{i s}^{\prime}\right\|_{\infty}+\left\|W_{i s}^{\prime}\right\|_{\infty}+\left\|s U_{i s}\right\|_{\infty}+\left\|s W_{i s}\right\|_{\infty}+\left\|f_{1}\right\|_{\infty}+\left\|f_{3}\right\|_{\infty}\right) \\
& \quad+\left(\left\|U_{i s}^{\prime}\right\|_{L^{2}}+\left\|s U_{i s}\right\|_{L^{2}}+\left\|f_{1}\right\|_{L^{2}}+\left\|W_{i s}^{\prime}\right\|_{L^{2}}+\left\|s W_{i s}\right\|_{L^{2}}+\left\|f_{3}\right\|_{L^{2}}\right) .
\end{aligned}
$$

Here and below, the constants $c>0$ only depend on the given constants. Combined with equation (2.8), Lemma 3.3 of [7] shows that

$$
\left\|s U_{i s}\right\|_{\infty},\left\|U_{i s}^{\prime}\right\|_{\infty} \leq c\left(\left\|f_{1}\right\|_{H^{1}}+\left\|f_{2}\right\|_{L_{2}}\right) .
$$

The maps $W_{i s}$ are treated similarly. Hence, (2.13) is true for $j \in\{I, I V, V\}$. In Cases II, III, and VI one has to add the norms $\left\|w_{1}\right\|_{L^{2}}$ and $\left\|w_{3}\right\|_{L^{2}}$ in (2.18), which can be absorbed using $|s| \geq 1$.

For completeness we also state the results from [19] and [12] for rational $d=c_{2} / c_{1}$. We write $d=o / e$ if $d=p / q$ for coprime integers with odd $p$ and even $q$, and analogously for the other two cases. Purely imaginary eigenvalues of $A_{j}$ exist if any only if

$$
\begin{aligned}
& \text { Case I : } \quad d=o / o, \quad \text { Case II : } d=o / e, \quad \text { Case III : } d \in \mathbb{Q} \text {, } \\
& \text { Case IV : } \quad d \in \mathbb{Q}, \quad \text { Case V: } d=e / o, \quad \text { Case VI }: \quad d=o / o \text {. }
\end{aligned}
$$

(Case III was treated in the proof of Proposition 2.2.)

\section{The CORE estimates And IRRATionality measures}

We now consider irrational $d=c_{2} / c_{1}>0$, so that $\Delta_{j}(i s) \neq 0$ for $s \in \mathbb{R} \backslash\{0\}$ by Proposition 2.2. Because of (2.13), we need lower bounds for $\left|\Delta_{j}(i s)\right|$ to control the growth of the resolvent of $A_{j}$ along $i \mathbb{R}$. Such bounds depend on the rate of simultaneous convergence of sequences $\left(s_{n} / c_{1}\right)$ and $\left(s_{n} / c_{2}\right)$ to the set of zeros of sine or cosine as $\left|s_{n}\right| \rightarrow \infty$ We are thus led to certain number theoretic properties of the ratio $d=c_{2} / c_{1}$.

Let $r>0$ be irrational. We define its irrationality measure $\mu(r)$ as the supremum of all $\theta>0$ such that

$$
\exists \text { infinitely many } p, q \in \mathbb{N} \text { with }\left|r-\frac{p}{q}\right|<q^{-\theta},
$$

see e.g. Appendix E in [10] or Chapter XI in [13]. Theorem 1 of [25] or Exercise E.1 in [10] give a formula for $\mu(r)$ in terms of the continued fraction of $r$. The larger $\mu(r)$ the better one can approximate $r$ by rationals and, as we see below, the faster $\Delta_{j}\left(i s_{n}\right)$ will tend to 0 for certain $\left|s_{n}\right| \rightarrow \infty$.

A famous result by Roth [22] says that $\mu(r)=2$ for algebraic $r$. One always has $\mu(r) \geq 2$, see Theorem 185 in [13]. It is known that $\mu(e)=2$, 
$\mu(\pi) \leq 7.6063$, and $\mu(\ln 2) \leq 3.5746$, for instance; see [9], [23], resp. [21]. There are numbers with $\mu(r)=\infty$, see Example 1 of [25] or $\S 11.7$ in [13]. One can construct $r$ with a prescribed irrationality measure due to Corollary 4 of [25]. We have $\mu(r)=2$ for a.e. $r$ by Theorem E.3 in [10].

However, the eigenvalue results (2.19) indicate that maybe one should not look for approximations by any rationals, but only by those in one of the classes odd/odd, odd/even, or even/odd. We thus define the restricted irrationality measures $\mu_{o o}(r), \mu_{o e}(r)$, and $\mu_{e o}(r)$ as the supremum of all $\theta>0$ satisfying (3.1) with $p / q=o / o, p / q=o / e$, respectively $p / q=e / o$. Clearly, these numbers are less or equal $\mu(r)$, and at least one of them has to be equal to $\mu(r)$. It is known that $\mu_{o o}(r), \mu_{o e}(r), \mu_{e o}(r) \geq 2$, and hence they are all equal to $\mu(r)$ if $\mu(r)=2$, see [24] or Theorem II in [17].

The continued fraction $\left[a_{0}, a_{1}, \ldots\right]$ of $r$ allows to relate these numbers. We first recall that only the convergents of $r$ can approach it faster than quadratically, see Theorem 184 in [13]. If all quotients $a_{n}$ are even integers, then the convergents belong to only two of the classes o/o, o/e and $e / o$ (determined by initial $a_{n}$ ), see Lemmas 1 and 2 of [17]. So, if e.g. $o / o$ is left out and $\mu(r)>2$, then $\mu_{o o}(r)=2$ and one of the other two classes is equal to $\mu(r)>2$. We set

$$
\begin{aligned}
& \text { Case I : } \quad \mu_{I}=\mu_{o o}(d), \quad \text { Case II : } \mu_{I I}=\mu_{o e}(d) \text {, } \\
& \text { Case III : } \mu_{I I I}=\mu(d), \quad \text { Case IV : } \mu_{I V}=\mu(d) \text {, } \\
& \text { Case V: } \quad \mu_{V}=\mu_{e o}(d), \quad \text { Case VI }: \mu_{V I}=\mu_{o o}(d) \text {. }
\end{aligned}
$$

The next lemma contains the core estimate to treat the case of irrational ratios $d$. It also shows that this estimate is almost optimal.

Lemma 3.1. Let $j \in J, d=c_{2} / c_{1}$ be irrational, and $\mu_{j}$ in (3.2) be finite. Take any $\eta>0$. Then the following assertions are true.

a) There exists a constant $c_{\eta}>0$ such that

$$
\left|\Delta_{j}(i s)\right| \geq \frac{c_{\eta}}{|s|^{\left(2 \mu_{j}-2\right)+\eta}}
$$

for all $s \in \mathbb{R}$ with $|s| \geq 1$.

b) There are numbers $\tilde{s}_{n} \in[1, \infty)$ with $\tilde{s}_{n} \rightarrow \infty$ and a constant $\tilde{c}>0$ with

$$
\left|\Delta_{j}\left(i \tilde{s}_{n}\right)\right| \leq \frac{\tilde{c}}{|s|^{\left(2 \mu_{j}-2\right)-\eta}}
$$

for all $n \in \mathbb{N}$. In (3.4) we can take $\eta=0$ if $\mu_{j}=2$.

Proof. We do not relabel subsequences of $\left(s_{n}\right)$, and write $\kappa$ or $\kappa_{l}$ for positive constants only depending on the given numbers $T_{j}, m_{j}$, and $k$.

1a) For $\lambda=i s$ and $s \in \mathbb{R}$, we can rewrite the maps $\Delta_{j}$ from (2.12) as

$$
\begin{aligned}
\Delta_{I}(i s) & =a_{1} a_{2} k \cos \left(\frac{s}{c_{1}}\right) \cos \left(\frac{s}{c_{2}}\right)+i\left[a_{2} \sin \left(\frac{s}{c_{1}}\right) \cos \left(\frac{s}{c_{2}}\right)+a_{1} \cos \left(\frac{s}{c_{1}}\right) \sin \left(\frac{s}{c_{2}}\right)\right], \\
\Delta_{I I}(i s) & =-a_{2} \sin \left(\frac{s}{c_{1}}\right) \sin \left(\frac{s}{c_{2}}\right)+a_{1} \cos \left(\frac{s}{c_{1}}\right) \cos \left(\frac{s}{c_{2}}\right)+i a_{1} a_{2} k \cos \left(\frac{s}{c_{1}}\right) \sin \left(\frac{s}{c_{2}}\right), \\
\Delta_{I I I}(i s) & =-a_{1} a_{2} k \sin \left(\frac{s}{c_{1}}\right) \sin \left(\frac{s}{c_{2}}\right)+i\left[a_{2} \cos \left(\frac{s}{c_{1}}\right) \sin \left(\frac{s}{c_{2}}\right)+a_{1} \sin \left(\frac{s}{c_{1}}\right) \cos \left(\frac{s}{c_{2}}\right)\right] . \\
\Delta_{I V}(i s) & =-k \sin \left(\frac{s}{c_{1}}\right) \sin \left(\frac{s}{c_{2}}\right)+i\left[a_{1} \cos \left(\frac{s}{c_{1}}\right) \sin \left(\frac{s}{c_{2}}\right)+a_{2} \sin \left(\frac{s}{c_{1}}\right) \cos \left(\frac{s}{c_{2}}\right)\right], \\
\Delta_{V}(i s) & =a_{1} \cos \left(\frac{s}{c_{1}}\right) \cos \left(\frac{s}{c_{2}}\right)-a_{2} \sin \left(\frac{s}{c_{1}}\right) \sin \left(\frac{s}{c_{2}}\right)+i k \sin \left(\frac{s}{c_{1}}\right) \cos \left(\frac{s}{c_{2}}\right), \quad(3.5) \\
\Delta_{V I}(i s) & =k \cos \left(\frac{s}{c_{1}}\right) \cos \left(\frac{s}{c_{2}}\right)+i\left[a_{1} \sin \left(\frac{s}{c_{1}}\right) \cos \left(\frac{s}{c_{2}}\right)+a_{2} \cos \left(\frac{s}{c_{1}}\right) \sin \left(\frac{s}{c_{2}}\right)\right] .
\end{aligned}
$$


Suppose that condition (3.3) does not hold. Then there exists a number $\bar{\eta} \in(0,1 / 3)$ and a sequence $\left(s_{n}\right)$ in $\mathbb{R}$ with $\left|s_{n}\right| \geq 1$ and $\left|s_{n}\right| \rightarrow \infty$ such that

$$
\left|\Delta_{j}\left(i s_{n}\right)\right| \leq\left|s_{n}\right|^{-\left(2 \mu_{j}-2\right)-3 \bar{\eta}}
$$

for all $n \in \mathbb{N}$ and some $j$.

We write $\Delta_{j}(i s)=\Delta_{j}^{(1)}(i s)+\Delta_{j}^{(2)}(i s)$, where $\Delta_{j}^{(1)}(i s)$ is the real part of $\Delta_{j}(i s)$ if $j \in\{I, I I I, I V, V I\}$ and the imaginary part if $j \in\{I I, V\}$. In each case, the formulas (3.5) yield the expression

$$
\Delta_{j}^{(1)}\left(i s_{n}\right)= \pm \kappa_{1} \varphi_{j}\left(s_{n} / c_{1}\right) \psi_{j}\left(s_{n} / c_{2}\right),
$$

for functions $\varphi_{j}, \psi_{j} \in\{\sin , \cos \}$ and a non-specified sign. Estimate (3.6) then implies that at least (a subsequence of) one of the sequences $\left(s_{n} / c_{1}\right)$ and $\left(s_{n} / c_{2}\right)$ approaches the set of zeros $N(1, j)$ of $\varphi_{j}$, respectively $N(2, j)$ of $\psi_{j}$. Here we have

$$
\begin{array}{rlrlrl} 
& \text { Case I : } & N(1, I) & =\pi \mathbb{Z}+\frac{\pi}{2}, & N(2, I) & =\pi \mathbb{Z}+\frac{\pi}{2}, \\
& \text { Case II : } & N(1, I I) & =\pi \mathbb{Z}+\frac{\pi}{2}, & N(2, I I) & =\pi \mathbb{Z}, \\
& \text { Case III : } & N(1, I I I) & =\pi \mathbb{Z}, & N(2, I I I) & =\pi \mathbb{Z}, \\
\text { Case IV : } & N(1, I V) & =\pi \mathbb{Z}, & N(2, I V) & =\pi \mathbb{Z}, \\
\text { Case V : } & N(1, V) & =\pi \mathbb{Z}, & N(2, V) & =\pi \mathbb{Z}+\frac{\pi}{2}, \\
\text { Case VI : } & N(1, V I) & =\pi \mathbb{Z}+\frac{\pi}{2}, & N(2, V I) & =\pi \mathbb{Z}+\frac{\pi}{2} .
\end{array}
$$

We now look at $\Delta_{j}^{(2)}\left(i s_{n}\right)$ which is in all cases of the form

$$
\Delta_{j}^{(2)}\left(i s_{n}\right)=\tau_{j}^{1} \kappa_{2} \tilde{\varphi}_{j}\left(s_{n} / c_{1}\right) \psi_{j}\left(s_{n} / c_{2}\right)+\tau_{j}^{2} \kappa_{3} \varphi_{j}\left(s_{n} / c_{1}\right) \tilde{\psi}_{j}\left(s_{n} / c_{2}\right),
$$

where $\tau_{j}^{l} \in\{1,-1\}$, as well as $\tilde{\varphi}_{j}=\sin$ if $\varphi_{j}=\cos$ and analogously in the other cases. If $\left(\varphi_{j}\left(s_{n} / c_{1}\right)\right)$ tends to 0 , the second summand in $\Delta_{j}^{(2)}\left(i s_{n}\right)$ vanishes and the numbers $\left|\tilde{\varphi}_{j}\left(s_{n} / c_{1}\right)\right|$ converge to 1 as $n \rightarrow \infty$. Therefore also $\psi_{j}\left(s_{n} / c_{2}\right)$ has to approach $N(2, j)$. The roles of $\varphi_{j}$ and $\psi_{j}$ can be interchanged here. Hence, $\varphi_{j}\left(s_{n} / c_{1}\right)$ and $\psi_{j}\left(s_{n} / c_{2}\right)$ tend to 0 as $n \rightarrow \infty$.

We can thus find non-zero integers $k_{n}$ and $l_{n}$ satisfying $\left|k_{n}\right|,\left|l_{n}\right| \rightarrow \infty$ and null sequences $\left(\delta_{n}\right)$ and $\left(\epsilon_{n}\right)$ bounded by $\pi / 2$ such that

$$
\frac{s_{n}}{c_{1}}=k_{n} \pi+\xi_{j} \frac{\pi}{2}+\delta_{n} \quad \text { and } \quad \frac{s_{n}}{c_{2}}=l_{n} \pi+\zeta_{j} \frac{\pi}{2}+\epsilon_{n}
$$

for all $n \in \mathbb{N}$. Here we have set

$$
\begin{aligned}
& \text { Case I : } \quad \xi_{I}=1, \quad \zeta_{I}=1, \quad \text { Case II : } \quad \xi_{I I}=1, \quad \zeta_{I I}=0, \\
& \text { Case III : } \quad \xi_{I I I}=0, \quad \zeta_{I I I}=0, \quad \text { Case IV }: \quad \xi_{I V}=0, \quad \zeta_{I V}=0, \\
& \text { Case V: } \quad \xi_{V}=0, \quad \zeta_{V}=1, \quad \text { Case VI }: \quad \xi_{V I}=1, \quad \zeta_{V I}=1 .
\end{aligned}
$$

It follows

$$
\begin{aligned}
c_{1} k_{n} \pi+c_{1} \xi_{j} \frac{\pi}{2}+c_{1} \delta_{n} & =c_{2} l_{n} \pi+c_{2} \zeta_{j} \frac{\pi}{2}+c_{2} \epsilon_{n}, \\
\frac{2 k_{n}+\xi_{j}}{2 l_{n}+\zeta_{j}}-d & =\frac{2}{\pi\left(2 l_{n}+\zeta_{j}\right)}\left(d \epsilon_{n}-\delta_{n}\right) .
\end{aligned}
$$

The assumption on $d$ now implies that

$$
\left|\frac{2 k_{n}+\xi_{j}}{2 l_{n}+\zeta_{j}}-d\right| \geq \frac{1}{\left|2 l_{n}+\zeta_{j}\right|^{\mu_{j}+\bar{\eta}}}
$$


for all $n \geq n_{\eta}$ and some index $n_{\eta} \in \mathbb{N}$, cf. (3.1), (3.2) and (3.10). After dropping finitely many members of the sequences, we then obtain the inequality

$$
\left|d \epsilon_{n}-\delta_{n}\right| \geq \frac{\bar{c}}{\left|l_{n}\right|^{\mu_{j}-1+\bar{\eta}}}
$$

for all $n \in \mathbb{N}, j \in J$, and a constant $\bar{c}>0$.

c) We claim that the sequences $\left(\delta_{n}\right)$ and $\left(\epsilon_{n}\right)$ satisfy the estimates

$$
\begin{gathered}
0<\liminf _{n \rightarrow \infty} \frac{\left|\epsilon_{n}\right|}{\left|\delta_{n}\right|} \leq \limsup _{n \rightarrow \infty} \frac{\left|\epsilon_{n}\right|}{\left|\delta_{n}\right|}<\infty, \\
\left|\epsilon_{n}\right|,\left|\delta_{n}\right| \geq \frac{\bar{c}^{\prime}}{\left|l_{n}\right|^{\mu_{j}-1+\bar{\eta}}}
\end{gathered}
$$

for a constant $\bar{c}^{\prime}>0$ and all $n \in \mathbb{N}$. We suppose that this claim was wrong.

First, there could be a subsequence such that $\lim _{n \rightarrow \infty} \frac{\left|\epsilon_{n}\right|}{\left|\delta_{n}\right|}=0$. Because of (3.11), the numbers $\delta_{n}$ then have to satisfy (3.13) for all sufficently large $n \in \mathbb{N}$ and some $\bar{c}^{\prime}>0$. Observe that $\left|\varphi_{j}\left(s_{n} / c_{1}\right)\right| \geq\left|\delta_{n}\right| / 2$ and $\left|\psi_{j}\left(s_{n} / c_{2}\right)\right| \leq\left|\epsilon_{n}\right|$ for all sufficiently large $n$ by (3.9), (3.10) and the Taylor series of sin and $\cos$ at their zeros. Similarly, the numbers $\left|\tilde{\varphi}_{j}\left(s_{n} / c_{1}\right)\right|$ and $\left|\tilde{\psi}_{j}\left(s_{n} / c_{2}\right)\right|$ tend to 1 as $n \rightarrow \infty$ and are thus contained in $[1 / 2,1]$ starting from some index $n_{0}$. We now use these facts and (3.9) to infer from (3.8) the lower bound

$$
\left|\Delta_{j}\left(i s_{n}\right)\right| \geq\left|\Delta_{j}^{(2)}\left(i s_{n}\right)\right| \geq \frac{\kappa_{3}}{4}\left|\delta_{n}\right|-\kappa_{2}\left|\epsilon_{n}\right| \geq \frac{\kappa_{3}}{8}\left|\delta_{n}\right| \geq \frac{\kappa_{3} \bar{c}^{\prime}}{8\left|l_{n}\right|^{\mu_{j}-1+\bar{\eta}}} \geq \frac{\kappa \bar{c}^{\prime}}{\left|s_{n}\right|^{\mu_{j}-1+\bar{\eta}}}
$$

for all sufficiently large $n$. But this inequality contradicts (3.6).

Second, if $\lim _{n \rightarrow \infty} \frac{\left|\epsilon_{n}\right|}{\left|\delta_{n}\right|}=\infty$ was true for a subsequence, we infer a contradiction in a similar way. Hence, the relations (3.12) are fulfilled, and then (3.11) implies the inequality (3.13).

d) As we see in step 2$)$, in $\Delta_{j}^{(2)}\left(i s_{n}\right)$ cancellations may occur. So we look at the term $\Delta_{j}^{(1)}\left(i s_{n}\right)$. Since the arguments approach the zero set of the functions $\varphi_{j}$ and $\psi_{j}$, from (3.7) and (3.13) we derive the lower bound

$$
\left|\Delta_{j}\left(i s_{n}\right)\right| \geq\left|\Delta_{j}^{(1)}\left(i s_{n}\right)\right| \geq \frac{\kappa_{1}}{4}\left|\delta_{n}\right|\left|\epsilon_{n}\right| \geq \frac{\kappa_{1}\left(\bar{c}^{\prime}\right)^{2}}{4\left|l_{n}\right|^{2 \mu_{j}-2+2 \bar{\eta}}} \geq \frac{\kappa\left(\bar{c}^{\prime}\right)^{2}}{\left|s_{n}\right|^{2 \mu_{j}-2+2 \bar{\eta}}}
$$

as above for all $n \in \mathbb{N}$. But this inequality cannot be true because of (3.6). So assertion (3.3) is shown.

2a) By the assumption on $d$, for all $\eta>0$ and $j \in J$ there are $k_{n}, l_{n} \in \mathbb{N}$ such that

$$
\left|d_{n}\right| \leq \frac{1}{\left|l_{n}\right|^{\mu_{j}-\eta / 2}}, \quad \text { where } \quad d_{n}:=\frac{2 k_{n}+\xi_{j}}{2 l_{n}+\zeta_{j}}-d
$$

for all $n \in \mathbb{N}$. Due to [24] we can put here $\eta=0$ if $\mu_{j}(d)=2$.

To indicate the cases, we now denote the (positive) prefactors $\kappa_{2}$ and $\kappa_{3}$ of $\Delta_{j}^{(2)}(i s)$ in (3.8) by $\alpha_{j}$ and $\beta_{j}$, respectively. (Only $\alpha_{I I}$ and $\beta_{V}$ differ from the corresponding factors in (3.5), which are equal to $-\alpha_{I I}$ and $-\beta_{V}$, respectively.) Recall the numbers $\xi_{j}$ and $\zeta_{j}$ from (3.10). We set

$$
b_{j}:=d+\frac{\alpha_{j}}{\beta_{j}}, \quad \delta_{n}:=\left(\frac{d}{b_{j}}-1\right) \frac{\pi}{2} d_{n}\left(2 l_{n}+\zeta_{j}\right), \quad \epsilon_{n}:=\frac{\pi}{2 b_{j}} d_{n}\left(2 l_{n}+\zeta_{j}\right)
$$


for $n \in \mathbb{N}$. By the estimate (3.14) there exists a constant $\tilde{c}>0$ with

$$
\left|\delta_{n}\right|,\left|\epsilon_{n}\right| \leq \frac{\tilde{c}}{\left|l_{n}\right|^{\mu_{j}-1-\eta / 2}}
$$

for every $n \in \mathbb{N}$, so that $\left|\delta_{n}\right|,\left|\epsilon_{n}\right| \leq \pi / 2$ for all large $n$. Following (3.9), we next define the numbers

$$
\tilde{s}_{n}:=c_{1} k_{n} \pi+c_{1} \xi_{j} \frac{\pi}{2}+c_{1} \delta_{n}
$$

Equations (3.15) then yield the formulas

$$
\tilde{s}_{n}=c_{2} l_{n} \pi+c_{2} \zeta_{j} \frac{\pi}{2}+c_{2} \epsilon_{n} \quad \text { and } \quad \alpha_{j} \epsilon_{n}+\beta_{j} \delta_{n}=0
$$

for all $n \in \mathbb{N}$.

b) As in part 1c) we estimate

$$
\left|\Delta_{j}^{(1)}\left(i \tilde{s}_{n}\right)\right| \leq \kappa_{1}\left|\varphi_{j}\left(\tilde{s}_{n} / c_{1}\right)\right|\left|\psi_{j}\left(\tilde{s}_{n} / c_{2}\right)\right| \leq \kappa_{1}\left|\delta_{n}\right|\left|\epsilon_{n}\right| \leq \frac{\kappa_{1} \tilde{c}^{2}}{\left|l_{n}\right|^{2 \mu_{j}-2-\eta}},
$$

for all $n \in \mathbb{N}$, using $(3.7),(3.17),(3.18)$ and (3.16). In $\Delta_{j}^{(2)}\left(i \tilde{s}_{n}\right)$ from (3.8) our construction will lead to a cancellation. Set

$$
\sigma_{n}^{(1)}:=\left\{\begin{array}{ll}
1, & k_{n} \text { even, } \\
-1, & k_{n} \text { odd },
\end{array} \sigma_{n}^{(2)}:=\left\{\begin{array}{ll}
1, & l_{n} \text { even, } \\
-1, & l_{n} \text { odd, }
\end{array} \quad \rho_{j}:= \begin{cases}1, & j \in\{I I I, I V\}, \\
-1, & \text { else },\end{cases}\right.\right.
$$

for $n \in \mathbb{N}$. We insert (3.17), (3.18) and (3.10) into the respective formulas in (3.5). Applying carefully the translation rules for sine and cosine, we arrive at the expression

$$
\Delta_{j}^{(2)}\left(i \tilde{s}_{n}\right)=\rho_{j} \sigma_{n}^{(1)} \sigma_{n}^{(2)}\left[\alpha_{j} \cos \left(\delta_{n}\right) \sin \left(\epsilon_{n}\right)+\beta_{j} \sin \left(\delta_{n}\right) \cos \left(\epsilon_{n}\right)\right] .
$$

The power series for sine and cosine, (3.18), and (3.16) thus imply

$$
\left|\Delta_{j}^{(2)}\left(i \tilde{s}_{n}\right)\right| \leq\left|\alpha_{j} \epsilon_{n}+\beta_{j} \delta_{n}\right|+O\left(\left|\delta_{n}\right|^{3}+\left|\epsilon_{n}\right|^{3}\right)=O\left(\left|l_{n}\right|^{-3 \mu_{j}+3+3 \eta / 2}\right) .
$$

So the term (3.19) dominates in $\Delta\left(i \tilde{s}_{n}\right)=\Delta_{j}^{(1)}\left(i \tilde{s}_{n}\right)+\Delta_{j}^{(2)}\left(i \tilde{s}_{n}\right)$ and yields assertion (3.4).

\section{MAIn RESUlts}

Lemma 3.1 and Proposition 2.2 imply the crucial resolvent estimates. Throughout we use the standard norm of $\mathcal{H}$.

Proposition 4.1. Let $j \in J, d=c_{2} / c_{1}$ be irrational, and $\mu_{j}$ in (3.2) be finite. Take any $\eta>0$. Then the following asserstions are true.

a) There exists a constant $C_{\eta}>0$ such that

$$
\|R(i s, A)\| \leq C_{\eta}|s|^{\left(2 \mu_{j}-2\right)+\eta}
$$

for all $s \in \mathbb{R}$ with $|s| \geq 1$.

b) There are numbers $\tilde{s}_{n} \in[1, \infty)$ with $\tilde{s}_{n} \rightarrow \infty$ and a constant $\tilde{C}>0$ with

$$
\left\|R\left(i \tilde{s}_{n}, A\right)\right\| \geq \tilde{C}|s|^{\left(2 \mu_{j}-2\right)-\eta}
$$

for all $n \in \mathbb{N}$. In $(4.2)$ we can take $\eta=0$ if $\mu_{j}=2$. 
Proof. The first assertion follows directly from (2.13) and (3.3). For the lower estimate, we take the numbers $\tilde{s}_{n} \rightarrow \infty$ from Lemma 3.1. Let $n \in \mathbb{N}$. To bound the norm of the resolvent, we use the functions $\widetilde{\chi}_{n}:[-1,0] \rightarrow \mathbb{R} ; \quad \widetilde{\chi}_{n}(x)=\left\{\begin{array}{ll}\cos \left(\tilde{s}_{n} x / c_{1}\right), & j \in\{I, I I, I I I\}, \\ \sin \left(\tilde{s}_{n} x / c_{1}\right), & j \in\{I V, V, V I\},\end{array} \quad \chi_{n}=\left\|\widetilde{\chi}_{n}\right\|_{L_{2}}^{-1} \widetilde{\chi}_{n}\right.$ and write $w^{n}=R\left(i \tilde{s}_{n}, A\right)\left(0, \chi_{n}, 0,0\right)$. Since $\left\|\chi_{n}\right\|_{L^{2}}=1$, it follows

$$
\left\|R\left(i \tilde{s}_{n}, A\right)\right\| \geq\left\|w^{n}\right\|_{\mathcal{H}} \geq\left\|w_{2}^{n}\right\|_{L^{2}} .
$$

In each case, formulas (2.9) and (2.8) yield

$$
w_{2}^{n}(x)=i \tilde{s}_{n} w_{1}^{n}(x)=i \tilde{s}_{n} \alpha\left(i \tilde{s}_{n}\right) g_{j}\left(\frac{i \tilde{s}_{n}}{c_{1}} x\right)-\frac{i}{c_{1}} \int_{-1}^{x} \sin \left(\frac{\tilde{s}_{n}}{c_{1}}(x-r)\right) \chi_{n}(r) \mathrm{d} r
$$

for $x \in[-1,0]$. By Lemma 3.3 of [7], the second summand is uniformly bounded by $c\left\|\chi_{n}\right\|_{L^{2}}=c$. The first one is given by

$$
G_{n, j}(x):=i \tilde{s}_{n} \alpha\left(i \tilde{s}_{n}\right) g_{j}\left(\frac{i \tilde{s}_{n}}{c_{1}} x\right)= \begin{cases}i \tilde{s}_{n} \alpha\left(i \tilde{s}_{n}\right) \cos \left(\frac{\tilde{s}_{n}}{c_{1}}(x+1)\right), & j \in\{I I I, V I\}, \\ -\tilde{s}_{n} \alpha\left(i \tilde{s}_{n}\right) \sin \left(\frac{\tilde{s}_{n}}{c_{1}}(x+1)\right), & \text { else. }\end{cases}
$$

Using the notation in (3.8), from formulas (2.10), (2.8), and (2.15) we derive the lower bound

$$
\begin{aligned}
\left|\tilde{s}_{n} \alpha\left(i \tilde{s}_{n}\right)\right| \geq & \frac{1}{\left|\Delta_{j}\left(i \tilde{s}_{n}\right)\right|}\left(\left|\tilde{\psi}_{j}\left(\frac{\tilde{s}_{n}}{c_{2}}\right) \frac{T_{1}}{c_{1}^{2}} \int_{-1}^{0} \cos \left(\frac{\tilde{s}_{n}}{c_{1}} r\right) \chi_{n}(r) \mathrm{d} r\right|\right. \\
& \left.-\left|a_{2} \psi_{j}\left(\frac{\tilde{s}_{n}}{c_{2}}\right)\left[\frac{-i}{c_{1}} \int_{-1}^{0} \sin \left(\frac{\tilde{s}_{n}}{c_{1}} r\right) \chi_{n}(r) \mathrm{d} r+\frac{k T_{1}}{c_{1}^{2}} \int_{-1}^{0} \cos \left(\frac{\tilde{s}_{n}}{c_{1}} r\right) \chi_{n}(r) \mathrm{d} r\right]\right|\right)
\end{aligned}
$$

for $j \in\{I, I I, I I I\}$ and $x \in[-1,0]$; whereas (2.11), (2.8), and (2.15) yield

$$
\begin{aligned}
\left|\tilde{s}_{n} \alpha\left(i \tilde{s}_{n}\right)\right| \geq & \frac{1}{\left|\Delta_{j}\left(i \tilde{s}_{n}\right)\right|}\left(\mid a_{2} \tilde{\psi}_{j}\left(\frac{\tilde{s}_{n}}{c_{2}}\right) \frac{-i}{c_{1}} \int_{-1}^{0} \sin \left(\frac{\tilde{s}_{n}}{c_{1}} r\right) \chi_{n}(r) \mathrm{d} r\right. \\
& \left.-\left|\psi_{j}\left(\frac{\tilde{s}_{n}}{c_{2}}\right)\left[\frac{T_{1}}{c_{1}^{2}} \int_{-1}^{0} \cos \left(\frac{\tilde{s}_{n}}{c_{1}} r\right) \chi_{n}(r) \mathrm{d} r-\frac{i k}{c_{1}} \int_{-1}^{0} \cos \left(\frac{\tilde{s}_{n}}{c_{1}} r\right) \chi_{n}(r) \mathrm{d} r\right]\right|\right)
\end{aligned}
$$

for $j \in\{I V, V, V I\}$.

In the above expresssions the integrals are bounded by $\left\|\chi_{n}\right\|_{L^{2}}=1$. As in part 1c) of the proof of Lemma 3.1 the numbers $\left|\tilde{\psi}_{j}\left(\frac{\tilde{s}_{n}}{c_{2}}\right)\right|$ tend to 1 and $\psi_{j}\left(\frac{\tilde{s}_{n}}{c_{2}}\right)$ to 0 as $n \rightarrow \infty$. We thus obtain an index $n_{0} \in \mathbb{N}$ such that

$$
\left|\tilde{s}_{n} \alpha\left(i \tilde{s}_{n}\right)\right| \geq \frac{\kappa}{\left|\Delta_{j}\left(i \tilde{s}_{n}\right)\right|\left\|\widetilde{\chi}_{n}\right\|_{L_{2}}} \int_{-1}^{0} \widetilde{\chi}_{n}(r)^{2} \mathrm{~d} r=\frac{\kappa}{\left|\Delta_{j}\left(i \tilde{s}_{n}\right)\right|}\left\|\widetilde{\chi}_{n}\right\|_{L_{2}}
$$

for all $n \geq n_{0}$. Moreover, a substitution and equation (3.17) lead to the inequality

$$
\begin{aligned}
\int_{-1}^{0} \cos ^{2}\left(\frac{\tilde{s}_{n}}{c_{1}} r\right) \mathrm{d} r & =\frac{c_{1}}{\tilde{s}_{n}} \int_{-\tilde{s}_{n} / c_{1}}^{0} \cos ^{2}(t) \mathrm{d} t \geq \frac{1}{\pi\left(k_{n}+1\right)} \int_{0}^{k_{n} \pi-\pi / 2} \cos ^{2}(t) \mathrm{d} t \\
& =\frac{2 k_{n}-1}{\pi\left(k_{n}+1\right)} \int_{0}^{\pi / 2} \cos ^{2}(t) \mathrm{d} t \geq \gamma>0,
\end{aligned}
$$

for some $\gamma>0$ and all $n$, and analogously

$$
\int_{-1}^{0} \cos ^{2}\left(\frac{\tilde{s}_{n}}{c_{1}}(r+1)\right) \mathrm{d} r \geq \widetilde{\gamma}>0 .
$$


Similar inequalities are valid if we replace here cos by sin. Summing up, we have shown that

$$
\left\|G_{n, j}\right\|_{L^{2}} \geq \frac{c}{\left|\Delta_{j}\left(i \tilde{s}_{n}\right)\right|}
$$

for all $j \in J$, all sufficiently large $n$ and a constant $c>0$ independent of $n$. Lemma 3.1 and (4.3) then imply (4.2).

The above estimates and the results [6] and [8] imply our main theorem. Recall that $2 \mu_{j}-2 \geq 2$ and that here equality holds for a.e. $d$ by Theorem E.3 in [10]. Moreover, $T_{I I I}(t) P_{I I I} w_{0}$ is constant in $x$ and affine in $t$.

Theorem 4.2. Let $j \in J, d=c_{2} / c_{1}$ be irrational, $\mu_{j}$ in (3.2) be finite, and $T_{j}(\cdot)$ the $C_{0}$-semigroup on $\mathcal{H}_{j}$ generated by $A_{j}$. Take any $\beta \in\left(0,\left(2 \mu_{j}-2\right)^{-1}\right)$ and $\gamma>\left(2 \mu_{j}-2\right)^{-1}$. There are constants $c_{\beta}, c>0$ and times $t_{n}=t_{n}(j) \geq 1$ tending to $\infty$ such that the following assertions hold.

a) Let $j \in\{I, I V, V\}$. The semigroups then satisfies

$$
\begin{aligned}
\left\|T_{j}(t) A_{j}^{-1}\right\| & \leq c_{\beta} t^{-\beta} \quad \text { for all } t \geq 1, \\
\left\|T_{j}\left(t_{n}\right) A_{j}^{-1}\right\| & \geq c t_{n}^{-\gamma} \quad \text { for all } n \in \mathbb{N} .
\end{aligned}
$$

b) Let $j \in\{I I, I I I, V I\}$ and $P_{j}$ be the spectral projectionm of $A_{j}$ for $\{0\}$ from Proposition 2.2. For $j \in\{I I, V I\}$ we obtain

$$
\begin{aligned}
\left\|T_{j}(t) w_{0}-P_{j} w_{0}\right\| & \leq c_{\beta} t^{-\beta}\left\|A_{j} w_{0}\right\| \quad \text { for all } t \geq 1, w_{0} \in D\left(A_{j}\right), \\
\left\|T_{j}\left(t_{n}\right)-P_{j}\right\|_{\mathcal{B}\left(D\left(A_{j}\right), \mathcal{H}_{j}\right)} & \geq c t_{n}^{-\gamma} \quad \text { for all } n \in \mathbb{N} .
\end{aligned}
$$

Moreover, we have

$$
\begin{aligned}
\left\|T_{I I I}(t) w_{0}-T_{I I I}(t) P_{I I I} w_{0}\right\| & \leq c_{\beta} t^{-\beta}\left\|A_{I I I}\left(I-P_{I I I}\right) w_{0}\right\|, \\
\left\|T_{I I I}\left(t_{n}\right)\left(I-P_{I I I}\right)\right\|_{\mathcal{B}\left(D\left(A_{I I I}\right), \mathcal{H}\right)} & \geq c t_{n}^{-\gamma},
\end{aligned}
$$

for alle $t \geq 1, w_{0} \in D\left(A_{I I I}\right)$, and $n \in \mathbb{N}$.

Proof. Let $j \in\{I, I V, V\}$. Part a) follows directly from Theorem 2.4 of [8] and Proposition 4.1 since $T_{j}(\cdot)$ is bounded on $\mathcal{H}_{j}$ and $\sigma\left(A_{j}\right) \cap i \mathbb{R}=\varnothing$ by Proposition 2.2.

Let $j \in\{I I, I I I, V I\}$. In these cases we use the restriction $A_{j}^{1}$ of $A_{j}$ to ker $P_{j} \subseteq \mathcal{H}_{j}$ which has the analogous properties as the generators in part a). The restriction $T_{j}^{1}(\cdot)$ of $T_{j}(\cdot)$ to $\operatorname{ker} P_{j}$ is generated by $A_{j}^{1}$, see Proposition 2.2, and hence satisfies estimates as in a). For $j \in\{I I, V I\}$ we further have $T_{j}(t) P_{j}=P_{j}$ and $A_{j} P_{j}=0$. The result follows.

In the above theorem we do not quite get the decay rate $\beta=\left(2 \mu_{j}-2\right)^{-1}$ since $\mu_{j}$ was defined by a supremum. At least for a.e. $d$ we can close this gap up to a logarithmic correction. Theorem 32 in [16] implies that for each $\eta>0$ we have

$$
\left|d-\frac{p}{q}\right| \geq \frac{1}{q^{2} \ln ^{1+\eta} q}
$$

for a.e. irrational $d>0$ and all rationals $p / q$ with $q \geq q_{\eta}$ for some $q_{\eta} \in \mathbb{N}$. On the other hand, for a.e. irrational $d$ one finds fractions $p_{n} / q_{n}$ with

$$
\left|d-\frac{p_{n}}{q_{n}}\right| \leq \frac{1}{q_{n}^{2} \ln q_{n}}
$$

for all $n \in \mathbb{N}$, so that (4.4) is essentially sharp. 
Theorem 4.3. For a.e. irrational $d=c_{2} / c_{1}$ and all $\epsilon>0$ there is a constant $c_{\epsilon}$ such that

$$
\begin{gathered}
\left\|T_{j}(t) A_{j}^{-1}\right\| \leq c_{\epsilon} \frac{\ln (t)^{1+\epsilon}}{\sqrt{t}}, \quad j \in\{I, I V, V\}, \\
\left\|T_{j}(t) w_{0}-P_{j} w_{0}\right\| \leq c_{\epsilon} \frac{\ln (t)^{1+\epsilon}}{\sqrt{t}}\left\|A_{j} w_{0}\right\|, \quad j \in\{I I, V I\}, \\
\left\|T_{I I I}(t) w_{0}-T_{I I I}(t) P_{I I I} w_{0}\right\| \leq c_{\epsilon} \frac{\ln (t)^{1+\epsilon}}{\sqrt{t}}\left\|A_{I I I}\left(I-P_{I I I}\right) w_{0}\right\|,
\end{gathered}
$$

for all $t \geq 1$ and $w_{0} \in D\left(A_{j}\right)$, where $P_{j}$ is the spectral projection of $A_{j}$ for $\{0\}$ from Proposition 2.2.

Proof. As in Lemma 3.1 one shows the lower bound

$$
\left|\Delta_{j}(i s)\right| \geq \frac{c_{\eta}}{s^{2} \ln (|s|)^{2+2 \eta}}
$$

for all $|s| \geq 1, j \in J$ and $\eta>0$. To this aim, we suppose that the opposite inequality is true for some $\bar{\eta}>0$ and a sequence $\left(s_{n}\right)$ with $\left|s_{n}\right| \rightarrow \infty$, cf. (3.6). We use (4.4) to replace (3.11) by

$$
\left|d \epsilon_{n}-\delta_{n}\right| \geq \frac{\bar{c}}{\left|l_{n}\right| \ln \left(\left|l_{n}\right|\right)^{1+\bar{\eta} / 2}}
$$

Arguing as in Lema 3.1, we then arrive at a contradiction and infer (4.5).

Proposition 2.2 now yields the estimate $\left\|R\left(i s, A_{j}\right)\right\| \leq c_{\eta}^{\prime} s^{2} \ln (|s|)^{2+2 \eta}$ for $|s| \geq 1$. Based on Theorem 1.3 of [5], we finally derive the assertions as in the previous theorem.

\section{REFERENCES}

[1] K. Ammari, M. Tucsnak and A. Henrot, Optimal location of the actuator for the pointwise stabilization of a string. C. R. Acad. Sci. Paris Sér. I Math. 330 (2000), $275-280$.

[2] N. Anantharaman and M. Léautaud, Sharp polynomial decay rates for the damped wave equation on the torus. Anal. PDE 7 (2014), 159-214.

[3] A. Bátkai, K.-J. Engel, J. Prüss and R. Schnaubelt, Polynomial stability of operator semigroups. Math. Nachr. 279 (2006), 1425-1440.

[4] C.J.K. Batty, A. Borichev and Y. Tomilov, $L^{p}$-tauberian theorems and $L^{p}$-rates for energy decay. J. Funct. Anal. 270 (2016), 1153-1201.

[5] C.J.K. Batty, R. Chill and Y. Tomilov, Fine scales of decay of operator semigroups. J. Eur. Math. Soc. 18 (2016), 853-929.

[6] C.J.K.Batty and T. Duyckaerts, Non-uniform stability for bounded semi-groups on Banach spaces. J. Evol. Equ. 8 (2008), 765-780.

[7] C.J.K. Batty, L. Paunonen and D. Seifert, Optimal energy decay in a one-dimensional coupled wave-heat system. J. Evol. Equ. 16 (2016), 649-664.

[8] A. Borichev and Y. Tomilov, Optimal polynomial decay of functions and operator semigroups. Math. Ann. 347 (2010), 455-478.

[9] J.M. Borwein and P.B. Borwein, Pi and the AGM. A Study in Analytic Number Theory and Computational Complexity. John Wiley, 1987.

[10] Y. Bugeaud, Distribution Modulo One and Diophantine Approximation. Cambridge University Press, 2012.

[11] G. Chen, M. Coleman and H.H. West, Pointwise stabilization in the middle of the span for second order systems nonuniform and uniform exponential decay of solutions. SIAM J. Appl. Math. 47 (1987), 751-780. 
[12] B.Z. Guo and W.D. Zhu, On the energy decay of two coupled strings through a joint damper. Journal of Sound and Vibration 203 (1997), 447-455.

[13] G.H. Hardy and E.M. Wright, An Introduction to the Theory of Numbers. 6th edition. Oxford University Press, 2008.

[14] S. Jaffard, M. Tucsnak, E. Zuazua, Singular internal stabilization of the wave equation. J. Differential Equations 145 (1998), 184-215.

[15] O. Kavian and Q. Zhang, Polynomial stabilization of solutions to a class of damped wave equations. Preprint (2017), arXiv:1703.01735.

[16] A.Ya. Khinchin, Continued Fractions. The University of Chicago Press, 1964.

[17] L. Kuipers and B. Meulenbeld, Some properties of continued fractions. Acta Math. 87 (1952). 1-12.

[18] G. Lebeau, Équation des ondes amorties. In: A. Boutet de Monvel and V. Marchenko (Eds.), "Algebraic and Geometric Methods in Mathematical Physics," Kluwer, 1996, pp. 73-109.

[19] K.-S. Liu, Energy decay problems in the design of a point stabilizer for coupled string vibrating systems. SIAM J. Control Optim. 26 (1988), 1348-1356.

[20] Z. Liu and B. Rao, Characterization of polynomial decay rate for the solution of linear evolution equation. Z. Angew. Math. Phys. 56 (2005), 630-644.

[21] R. Marcovecchio, The Rhin-Viola method for $\log 2$. Acta Arith. 139 (2009), 147-184.

[22] K.F. Roth, Rational approximations to algebraic numbers. Mathematika 2 (1955), $1-20$.

[23] V. Kh. Salikhov, On the irrationality measure of $\pi$. Russian Math. Surveys 63 (2008), 570-572.

[24] W.T. Scott, Approximation to real irrationals by certain classes of rational fractions. Bull. Amer. Math. Soc. 46 (1940), 124-129.

[25] J. Sondow, Irrationality measures, irrationality basis, and a theorem of Jarnik. Preprint, arXiv:math/0406300.

[26] M. Tucsnak, On the pointwise stabilization of a string. In: W. Desch, F. Kappel and K. Kunisch (Eds.), "Control and Estimation of Distributed Parameter Systems," Birkhäuser, 1998, pp. 287-295,

Ł. Rzepnicki, Faculty of Mathematics and Computer Science, Nicolaus CoPernicus University, Toruń, Poland.

E-mail address: keleb@mat.umk.pl

R. Schnaubelt, Department of Mathematics, Karlsruhe Institute of TechNOlogy, 76128 Karlsruhe, Germany.

E-mail address: schnaubelt@kit.edu 